\title{
Infalling groups and galaxy transformations in the cluster A2142
}

\author{
Maret Einasto $^{1}$, Boris Deshev ${ }^{1,2}$, Heidi Lietzen ${ }^{1}$, Rain Kipper ${ }^{1}$, Elmo Tempel ${ }^{1,3}$, Changbom Park ${ }^{4}$, Mirt Gramann ${ }^{1}$, \\ Pekka Heinämäki $^{5}$, Enn Saar ${ }^{1,6}$, and Jaan Einasto ${ }^{1,6,7}$ \\ 1 Tartu Observatory, Observatooriumi 1, 61602 Tõravere, Estonia \\ e-mail: maret.einasto@to.ee \\ 2 Institute of Physics, University of Tartu, W. Ostwaldi 1, 50411 Tartu, Estonia \\ 3 Leibniz-Institut für Astrophysik Potsdam (AIP), An der Sternwarte 16, 14482 Potsdam, Germany \\ ${ }^{4}$ School of Physics, Korea Institute for Advanced Study, 85 Hoegiro, Dong-Dae-Mun-Gu, 02455 Seoul, Korea \\ 5 Tuorla Observatory, University of Turku, Väisäläntie 20, 20014 Piikkiö, Finland \\ ${ }^{6}$ Estonian Academy of Sciences, Kohtu 6, 10130 Tallinn, Estonia \\ 7 ICRANet, Piazza della Repubblica 10, 65122 Pescara, Italy
}

Received 19 July 2017 / Accepted 12 November 2017

\begin{abstract}
Context. Superclusters of galaxies provide dynamical environments for the study of the formation and evolution of structures in the cosmic web from galaxies, to the richest galaxy clusters, and superclusters themselves.

Aims. We study galaxy populations and search for possible merging substructures in the rich galaxy cluster A2142 in the collapsing core of the supercluster SCl A2142, which may give rise to radio and X-ray structures in the cluster, and affect galaxy properties of this cluster.

Methods. We used normal mixture modelling to select substructure of the cluster A2142. We compared alignments of the cluster, its brightest galaxies (hereafter BCGs), subclusters, and supercluster axes. The projected phase space (PPS) diagram and clustercentric distributions are used to analyse the dynamics of the cluster and study the distribution of various galaxy populations in the cluster and subclusters.

Results. We find several infalling galaxy groups and subclusters. The cluster, supercluster, BCGs, and one infalling subcluster are all aligned. Their orientation is correlated with the alignment of the radio and X-ray haloes of the cluster. Galaxy populations in the main cluster and in the outskirts subclusters are different. Galaxies in the centre of the main cluster at the clustercentric distances $0.5 \mathrm{~h}^{-1} \mathrm{Mpc}\left(D_{\mathrm{c}} / R_{\mathrm{vir}}<0.5, R_{\text {vir }}=0.9 \mathrm{~h}^{-1} \mathrm{Mpc}\right)$ have older stellar populations (with the median age of 10-11 Gyr) than galaxies at larger clustercentric distances. Star-forming and recently quenched galaxies are located mostly at the clustercentric distances $D_{\mathrm{c}} \approx$ $1.8 \mathrm{~h}^{-1} \mathrm{Mpc}$, where subclusters fall into the cluster and the properties of galaxies change rapidly. In this region the median age of stellar populations of galaxies is about 2 Gyr. Galaxies in A2142 on average have higher stellar masses, lower star formation rates, and redder colours than galaxies in rich groups. The total mass in infalling groups and subclusters is $M \approx 6 \times 10^{14} \mathrm{~h}^{-1} M_{\odot}$, that is approximately half of the mass of the cluster. This mass is sufficient for the mass growth of the cluster from redshift $z=0.5$ (half-mass epoch) to the present.

Conclusions. Our analysis suggests that the cluster A2142 has formed as a result of past and present mergers and infallen groups, predominantly along the supercluster axis. Mergers cause complex radio and X-ray structure of the cluster and affect the properties of galaxies in the cluster, especially at the boundaries of the cluster in the infall region. Explaining the differences between galaxy populations, mass, and richness of A2142, and other groups and clusters may lead to better insight about the formation and evolution of rich galaxy clusters.
\end{abstract}

Key words. large-scale structure of Universe - galaxies: groups: general - galaxies: clusters: general - galaxies: clusters: individual: A2142

\section{Introduction}

The cosmic web, evolved from tiny density perturbations in the very early Universe, consists of galaxies and galaxy systems from the smallest groups to the richest superclusters, separated by voids (Jõeveer et al. 1978; Kofman \& Shandarin 1988).

Early studies of the cosmic web have already shown that galaxy superclusters have important morphological property: the most luminous galaxy clusters are typically located in the highdensity core regions of rich superclusters at the crossing of several cluster or galaxy filament chains (Jõeveer \& Einasto 1978; Jõeveer et al. 1978). These are locations in which rich galaxy clusters form via merging and accretion of smaller structures (galaxies and groups of galaxies) along filaments (Bond et al. 1996; van de Weygaert \& Schaap 2009; Suhhonenko et al. 2011; Kravtsov \& Borgani 2012, and references therein).

The high-density cores of rich superclusters are the largest objects in the Universe that may collapse now or during future evolution (Small et al. 1998; Reisenegger et al. 2000; Proust et al. 2006; Pearson et al. 2014; Gramann et al. 2015; O'Mill et al. 2015; Chon et al. 2015; Einasto et al. 2016). These cores form an evolving environment for the study of the properties of galaxies, groups, and clusters inside them. It is especially interesting to investigate rich galaxy clusters in the collapsing cores of superclusters, where we can study various processes and transformation of galaxies both in clusters and 
their outskirts, and in their larger scale environment. These processes are responsible for transformation of galaxies from mostly blue, star-forming field galaxies to red, quiescent cluster population (see Haines et al. 2015; Rhee et al. 2017, for a review and references).

Merging clusters in the collapsing cores of rich superclusters have been studied, for example, in the Shapley supercluster (Merluzzi et al. 2015) and in the A2199 supercluster (Song et al. 2017). One of such clusters is the very rich galaxy cluster A2142 embedded in the collapsing core of the supercluster SCl A2142 (Einasto et al. 2015; Gramann et al. 2015).

The structure of the central region of the cluster A2142 up to virial radius has been studied in detail in optical (Owers et al. 2011; Munari et al. 2014), radio (Govoni et al. 2010; Farnsworth et al. 2013; Venturi et al. 2017), and X-ray (also joint analysis with optical bands) wavelengths (Markevitch et al. 2000; Forman et al. 2001; Okabe \& Umetsu 2008; Rossetti et al. 2013; Eckert et al. 2014, 2017; Tchernin et al. 2016). These studies revealed that this cluster is a cold front cluster with signs of gas sloshing and giant multiple radio haloes that have been interpreted as signs of multiple mergers. Eckert et al. (2014, 2017) discovered a stripped galaxy group infalling into A2142. Rossetti et al. (2013) showed that in A2142 (merger induced) gas sloshing occurs at scales up to about $1 \mathrm{~h}^{-1} \mathrm{Mpc}$, which is much larger than typically in clusters and larger than obtained from simulations. A recent analysis of radio data of A2142 suggest several mergers and/or infalling galaxy groups in A2142 (Venturi et al. 2017). The cluster A2142 is unusually rich; it is, for example, twice as rich as the richest galaxy clusters in the Sloan Great Wall (SGW) approximately at the same distance (Einasto et al. 2010; Tempel et al. 2014b). Munari et al. (2016) showed that in simulations massive haloes have significantly lower amount of massive subhaloes in comparison with A2142.

In this paper we analyse the substructure and galaxy content of the cluster A2142 and its outskirts at clustercentric distances up to about $3 \mathrm{~h}^{-1} \mathrm{Mpc}$. We present an analysis of the full supercluster SCl A2142 in a forthcoming study. Our aim is to search for possible substructures that may give rise of the radio and X-ray structures of A2142 cluster and affect galaxy properties in the cluster. We determine substructures in the cluster and its outskirts and analyse their orientations. We study galaxy populations of the cluster and subclusters in the projected phase space (PPS) diagram and as a function of clustercentric distance. Recently, the analysis of the PPS diagram has become an important tool to study galaxy populations in galaxy groups, clusters, and multiclusters (Haines et al. 2015; Jaffé et al. 2015; Agulli et al. 2017; Paccagnella et al. 2017; Yoon et al. 2017; Rhee et al. 2017; Weinzirl et al. 2017). In the PPS diagram galaxies with different accretion histories populate different areas. This can be used to analyse dynamical properties of galaxies in clusters and outskirts and to compare galaxy populations in virialised and nonvirialised regions of the clusters. We also compare galaxy populations in A2142 and in other rich galaxy groups and clusters from the Sloan Digital Sky Survey.

We assume the standard cosmological parameters: the Hubble parameter $H_{0}=100 \mathrm{~h} \mathrm{~km} \mathrm{~s}^{-1} \mathrm{Mpc}^{-1}$, matter density $\Omega_{\mathrm{m}}=0.27$, and dark energy density $\Omega_{\Lambda}=0.73$ (Komatsu et al. 2011).

\section{Data}

\subsection{Supercluster, group, and filament data}

Our initial dataset is selected from the MAIN sample of the 10th data release of the Sloan Digital Sky Survey (SDSS)
(Aihara et al. 2011; Ahn et al. 2014). We used a spectroscopic galaxy sample with the apparent Galactic extinction corrected $r$ magnitudes $r \leq 17.77$ and redshifts $0.009 \leq z \leq 0.200$. We corrected the redshifts of galaxies for the motion relative to the cosmic microwave background and computed the comoving distances of galaxies (Martínez \& Saar 2002). Galaxies with unreliable parameters were removed from the sample as described in detail in Tempel et al. (2012, 2014b). The SDSS spectroscopic sample is incomplete because of fibre collisions; the smallest separation between spectroscopic fibres is 55", and approximately $6 \%$ of the potential targets for spectroscopy are without observed spectra because of this. Below we analyse the completeness of the data used in this paper due to fibre collisions.

The SDSS MAIN dataset was used to calculate the luminosity-density field and detect superclusters of galaxies in this field to find groups of galaxies with the friends-of-friends algorithm and to determine galaxy filaments by applying the Bisous process to the distribution of galaxies (Tempel et al. 2014a). The data from supercluster, group, and filament catalogues were then used to select galaxy group and filament information for the supercluster SCl A2142.

Supercluster catalogue and supercluster SCl A2142. Data on $\mathrm{SCl}$ A2142 are taken from the catalogue of galaxy superclusters in which superclusters were defined as extended connected volumes above a threshold density level 5.0 (in units of mean density, $\left.\ell_{\text {mean }}=1.65 \times 10^{-2} \frac{10^{10} \mathrm{~h}^{-2} L_{\odot}}{\left(\mathrm{h}^{-1} \mathrm{Mpc}\right)^{3}}\right)$ in the luminosity density field (Liivamägi et al. 2012).

The supercluster $\mathrm{SCl} \mathrm{A} 2142$ ( $\mathrm{SCl} 001$ in Liivamägi et al. 2012) at redshift $z \approx 0.09$ has over a thousand member galaxies; the total length of this supercluster, defined as the maximum distance between galaxy pairs in the supercluster, is $\approx 50 \mathrm{~h}^{-1} \mathrm{Mpc}$ (Liivamägi et al. 2012). This supercluster was recently described in Einasto et al. (2015) and Gramann et al. (2015) who showed that the high-density core of this supercluster, with a radius $\approx 13 \mathrm{~h}^{-1} \mathrm{Mpc}$ and mass of about $M \approx 4 \times 10^{15} \mathrm{~h}^{-1} M_{\odot}$, is already collapsing.

Galaxy groups in the supercluster SCl A2142. We selected galaxy groups belonging to $\mathrm{SCl} \mathrm{A} 2142$ from the group catalogue by Tempel et al. (2014b). In this catalogue galaxy groups were determined using the friends-of-friends cluster analysis method introduced in cosmology by Zeldovich et al. (1982) and Huchra \& Geller (1982). A galaxy belongs to a group of galaxies if this galaxy has at least one group member galaxy closer than a linking length. In a flux-limited sample the density of galaxies slowly decreases with distance. To take this selection effect into account properly when constructing a group catalogue from a flux-limited sample, the linking length was rescaled with distance, calibrating the scaling relation by observed groups. As a result, the maximum sizes in the sky projection and the velocity dispersions of Tempel et al. (2014b) groups are similar at all distances. The details about the data reduction, group finding procedure, and description of the group catalogue can be found in Tempel et al. (2014b).

$\mathrm{SCl}$ A2142 embeds 14 galaxy groups with at least 10 member galaxies. In the present study, we only used the data of the cluster A2142 (Gr3070 in the catalogue) (Table 1). The full supercluster $\mathrm{SCl}$ A2142 covers only a narrow distance interval $\left(\approx 255-275 \mathrm{~h}^{-1} \mathrm{Mpc}\right)$, and therefore we used data about the group from flux-limited sample (see also Einasto et al. 2015, for details of data selection). In Table 1 we also give data on A2142 components and subclusters, defined below in Sect. 3.1. Masses of the cluster A2142 and its components in Table 1 were calculated as described in Tempel et al. (2014b). These authors 


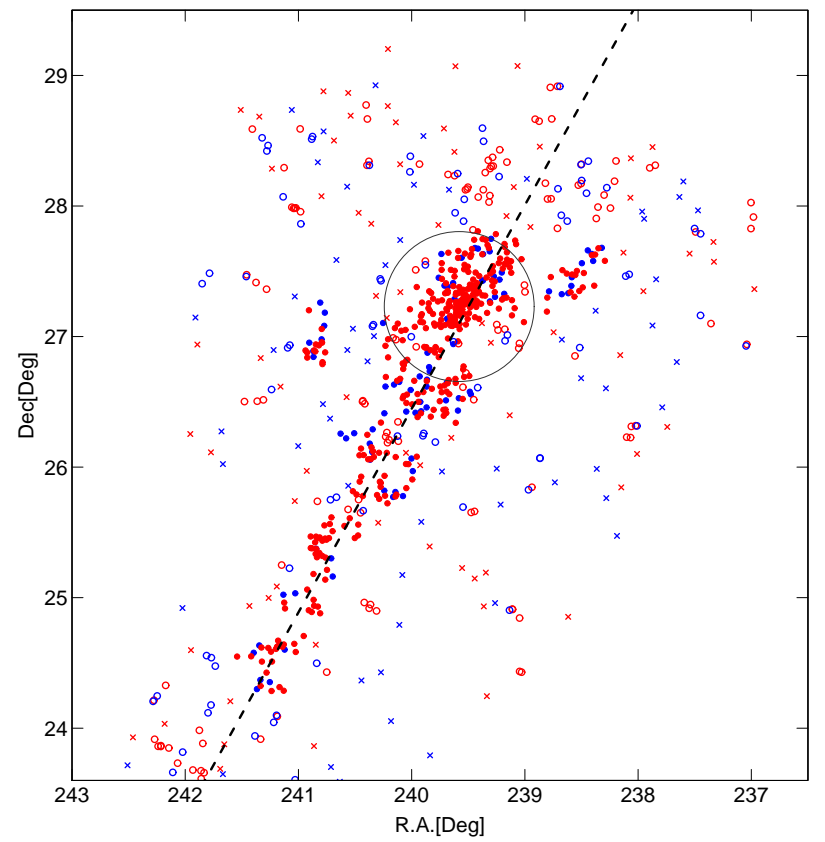

Fig. 1. Sky distribution of galaxies in SCl A2142. The red symbols show galaxies with old stellar populations $\left(D_{n}(4000) \geq 1.55\right)$, and the blue symbols denote galaxies with young stellar populations $\left(D_{n}(4000)<\right.$ 1.55). The filled circles correspond to galaxies in rich groups with at least 10 member galaxies, the empty circles indicate galaxies in poor groups with 2-9 galaxies, and the crosses denote single galaxies. The grey circle has a radius of about $3 \mathrm{~h}^{-1} \mathrm{Mpc}$ and denotes the region of the cluster A2142 and its outskirts. The dashed line shows the supercluster axis.

applied the virial theorem, assuming symmetry of galaxy velocity distribution and the Navarro-Frenk-White (NFW) density profile for galaxy distribution in the plane of the sky. For a detailed description of how the masses were calculated, we refer to Tempel et al. (2014b). The virial radius of the cluster, $R_{\mathrm{vir}}=0.9 \mathrm{~h}^{-1} \mathrm{Mpc}$. It is calculated as

$\frac{1}{R_{\mathrm{vir}}}=\frac{2}{\left(1+z_{\mathrm{m}}\right) n(n-1)} \sum_{i \neq j}^{n} \frac{1}{R_{i j}}$,

where $R_{i j}$ is the projected distance between galaxies in pairs in a group, $z_{\mathrm{m}}$ is the mean redshift of the group, and $n$ is the number of galaxies in a group (see Tempel et al. 2014b, for details). Figure 1 shows the sky distribution of galaxies in SCl A2142. We plot galaxies from rich and poor groups with filled and empty circles, correspondingly. Sky coordinates of galaxies from rich groups with at least 10 member galaxies have been used below to determine the orientation of the supercluster axis. We denote the region of the cluster A2142 and its outskirts analysed in this study.

Absolute magnitudes of galaxies are computed according to the formula

$M_{r}=m_{r}-25-5 \log _{10}\left(d_{\mathrm{L}}\right)-K$,

where $d_{\mathrm{L}}$ is the luminosity distance in units of $\mathrm{h}^{-1} \mathrm{Mpc}$ and $K$ is the $k+e$-correction. The $k$-corrections were calculated with the KCORRECT (v4_2) code (Blanton \& Roweis 2007) and the evolution corrections have been calibrated according to Blanton et al. (2003). Details about how the $k$ and $e$ corrections were applied can be found in Tempel et al. (2014b). Tempel et al. (2014b) used slightly smaller evolution corrections than Blanton et al. (2003). The value of $M_{\odot}=4.53$ (in $r$-filter).
Table 1. Data for the Abell cluster A2142 (Gr3070) components.

\begin{tabular}{rrrrrrr}
\hline \hline$(1)$ & $(2)$ & $(3)$ & $(4)$ & $(5)$ & $(6)$ & $(7)$ \\
\hline ID & $N_{\text {gal }}$ & $\begin{array}{r}\text { RA } \\
{[\mathrm{deg}]}\end{array}$ & $\begin{array}{r}\text { Dec } \\
{[\mathrm{deg}]}\end{array}$ & $\begin{array}{r}\text { Dist. } \\
{\left[\mathrm{Mpc} \mathrm{h}^{-1}\right]}\end{array}$ & $D_{n}(4000)$ \\
\hline$A 2142$ & 212 & 239.5 & 27.3 & 265 & 9.1 & 1.9 \\
$C 1$ & 135 & 239.6 & 27.2 & 266 & 7.1 & 1.9 \\
$C 2$ & 64 & 239.3 & 27.5 & 262 & 2.0 & \\
\hline$M 1$ & 22 & 239.3 & 27.3 & 257 & 0.9 & 1.9 \\
$M 2$ & 17 & 239.4 & 27.7 & 262 & 0.9 & 1.7 \\
$M 3$ & 24 & 239.2 & 27.6 & 262 & 1.5 & 1.8 \\
\hline$C 3$ & 8 & 239.8 & 26.9 & 255 & 1.5 & 1.9 \\
\hline
\end{tabular}

Notes. Columns are as follows: (1): Component ID (Einasto et al. 2015); (2): the number of galaxies in a component; (3-5) RA, Dec, and distance of a component centre; (6): the mass of a component, in units of $10^{14} \mathrm{~h}^{-1} M_{\odot}$; and (7): median value of the $D_{n}(4000)$ index (see Sect. 3.2).

At the distance of A2142, $\approx 265 \mathrm{~h}^{-1} \mathrm{Mpc}$, the sample is complete at the absolute magnitude limit $M_{r}=-19.6$ in units of mag $+5 \log _{10} h$. In our sample there is six galaxies fainter than this limit. In our study we used the full dataset of A2142 to determine the substructure in A2142, and for statistical analysis we used a magnitude-limited complete sample, excluding galaxies fainter than the completeness limit, $M_{r}=-19.6+5 \log _{10} h$. In addition, we checked the data in the region covered by the cluster A2412 (Gr3070) used in this paper and found that in this region no galaxy is missing because of the fibre collision effect.

Galaxy filaments. Galaxy filaments belonging to the supercluster SCl A2142 were extracted from the catalogue of galaxy filaments by Tempel et al. (2014a). This catalogue was built by applying the Bisous process to the distribution of galaxies (Tempel et al. 2016). Details about how the galaxy filaments in SCl A2142 were selected were described in Einasto et al. (2015). In the present study, we only used the data about filaments in the neighbourhood of the cluster A2142 to check for the filament membership of galaxies. Combining data from different datasets (supercluster, group, and filament catalogues) gives us more comprehensive information about the cluster and its outskirts. Catalogues of galaxy superclusters, groups, and filaments are available from the database of cosmology-related catalogues ${ }^{1}$.

\subsection{Galaxy populations}

Data about galaxies were downloaded from the SDSS DR10 web page $^{2}$ Galaxy magnitudes and Petrosian radii were taken from the SDSS spectroscopic and photometric data, correspondingly. We calculated galaxy colours as $(g-r)_{0}=M_{g}-M_{r}$. All magnitudes and colours correspond to the rest frame at redshift $z=0$. The concentration index of galaxies is calculated as the ratio of the Petrosian radii $R_{50}$ and $R_{90}: R_{50} / R_{90}$.

Galaxy stellar masses, star formation rates (SFRs), and $D_{n}(4000)$ index of galaxies are from the MPA-JHU spectroscopic catalogue (Tremonti et al. 2004; Brinchmann et al. 2004), from which the various properties of galaxies were obtained by fitting SDSS photometry and spectra with the stellar population synthesis models developed by Bruzual \& Charlot

\footnotetext{
1 http://cosmodb.to.ee/

2 http://skyserver.sdss3.org/dr10/en/help/browser/ browser.aspx
} 

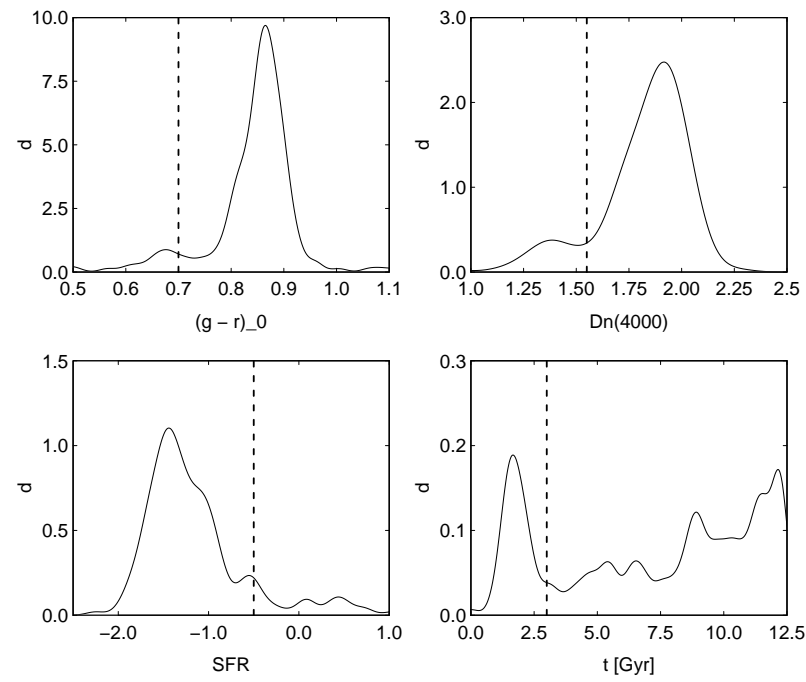

Fig. 2. Distribution of galaxy colours $(g-r)_{0}$ (upper left panel), $D_{n}(4000)$ index (upper right panel), star formation rates log SFR (lower left panel), and stellar ages $t$ (lower right panel) in the cluster A2142. The dashed lines show the parameter values used to divide galaxies into populations as described in the text.

(2003). The stellar masses of galaxies were derived as described by Kauffmann et al. (2003a). The SFRs were computed using the photometry and emission lines as described by Brinchmann et al. (2004) and Salim et al. (2007). The strength of the $D_{n}(4000)$ break (the ratio of the average flux densities in the band 4000-4100 $\AA$ and 3850-3950 $⿱ 亠 0.5 D_{n}(4000)$ index) is correlated with the time passed from the most recent star formation event and is defined as in Balogh et al. (1999). The $D_{n}(4000)$ index characterises star formation histories of galaxies.

The stellar velocity dispersions were obtained by fitting galaxies with the publicly available Gas AND Absorption Line Fitting (GANDALF; Sarzi et al. 2006) and penalised PiXel Fitting (pPXF; Cappellari \& Emsellem 2004). Stellar ages are from the Portsmouth group (Maraston et al. 2009).

In Fig. 2 we plot the distributions of galaxy colours $(g-r)_{0}$, $D_{n}(4000)$ index, star formation rates $\log S F R$, and stellar ages $t$ in the cluster A2142.

The value $(g-r)_{0}=0.7$ is used to separate red and blue galaxies, where red galaxies have $(g-r)_{0} \geq 0.7$. The percentage of red galaxies in A2142 is $f_{\text {red }}=0.92$.

The $D_{n}(4000)$ index was used to separate quiescent and starforming galaxies. We applied values for quiescent galaxies with old stellar populations as having $D_{n}(4000) \geq 1.55$ (89\% of all galaxies from the complete sample). Star-forming galaxies with young stellar populations have $D_{n}(4000)<1.55$. This limit was also used by Kauffmann et al. (2003b) and Haines et al. (2017b) to separate young and old galaxies in the SDSS survey.

We divided galaxies with old and young stellar populations by stellar age using the age limit $t=3$ Gyr. Galaxies with $t \geq$ 3 Gyr formed $77 \%$ of all galaxies. The SFR $\log S F R \leq-0.5$ corresponds to quiescent galaxies (88\% of galaxies). Actively star-forming galaxies are characterised by $\log S F R>-0.5$; this limit was also applied in Einasto et al. (2014).

We might use slightly higher values of the colour index $(g-r)_{0}$ and star formation rate $\log S F R$ to separate galaxy populations, this only changes the percentages of red and star-forming galaxies marginally. For example, if for red galaxies $(g-r)_{0} \geq$ 0.75 then the percentage of red galaxies in A2142 becomes

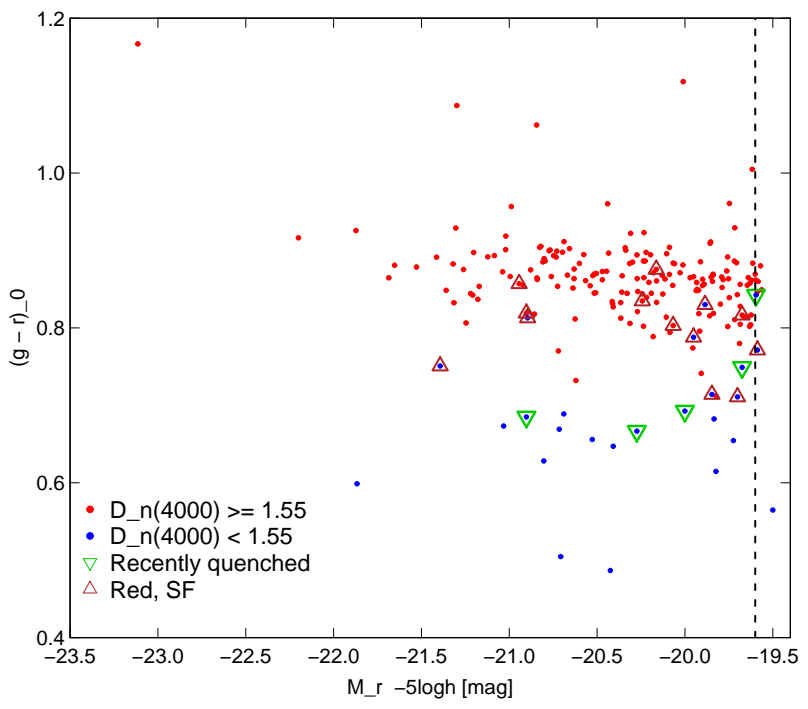

Fig. 3. Colour-magnitude diagram $(g-r)_{0}$ vs. $M_{r}$ for galaxies in the cluster A2142. The red filled circles correspond to the galaxies with old stellar populations $\left(D_{n}(4000) \geq 1.55\right)$ and the blue filled circles to the galaxies having young stellar populations with $D_{n}(4000)<1.55$. The green triangles show recently quenched galaxies with $D_{n}(4000) \leq 1.55$ and star formation rate $\log S F R<-0.5$. The red triangles indicate red, high SFR galaxies defined as $g-r \geq 0.7$, and $\log S F R \geq-0.5$. The dashed line shows the completeness limit $M_{r}=-19.6+5 \log _{10} h$.

$f_{\text {red }}=0.89$. Present values were used consistently with earlier studies (see, for example, Einasto et al. 2014; Haines et al. 2017b).

Some star-forming galaxies have red colours and are known as red star-forming galaxies. Also, there are galaxies with low SFRs but also low values of $D_{n}(4000)$ index, which suggest that they may be recently quenched. In our sample we define recently quenched galaxies as those with $D_{n}(4000) \leq 1.55$ and $\log S F R<-0.5$ (five galaxies). Red, high SFR galaxies are defined as galaxies with $(g-r)_{0} \geq 0.7$ and $\log S F R \geq-0.5$ (13 galaxies).

The A2142 colour-magnitude diagram is shown in Fig. 3. We plot galaxies with old and young stellar populations, recently quenched galaxies, and red, star-forming galaxies with different symbols. Here we show also the completeness limit in luminosity, $M_{r}=-19.6+5 \log _{10} h$. As seen also from this figure, most galaxies in A2142 are red, including two recently quenched galaxies. Among galaxies that are fainter than the completeness limit only one is blue.

\section{Analysis}

\subsection{Structure of the cluster A2142 and its outskirts}

The structure of the cluster A2142 has been analysed in Einasto et al. (2012) with a number of 1D, 2D, and 3D tests. Both 3D and 2D analyses revealed that A2142 has three components. The distribution of galaxy velocities in the components overlap, so that 1D methods do not find significant substructures in the cluster. The details of the tests can be found in Einasto et al. (2012). In the present study we focus on the 3D analysis of the cluster structure, following Einasto et al. (2015) who used multidimensional normal mixture modelling to separate the main A2142 cluster and its outskirts systems.

To search for components in A2142, mclust package for classification and clustering (Fraley \& Raftery 2006) from $R$, 
an open-source free statistical environment developed under the GNU GPL (Ihaka \& Gentleman 1996³) was applied. This package is based on the analysis of a finite mixture of distributions, in which each mixture component is taken to correspond to a different subgroup of the cluster. As an input for mclust we used the sky coordinates and velocity of the cluster A2142 member galaxies. The values of velocities were scaled to make them of the same order as the values of coordinates. The best solution for components found by mclust was chosen using the Bayesian information criterion (BIC). To test how the possible errors in the line-of-sight positions of galaxies affect the results of component analysis, the velocities of galaxies were shifted randomly 1000 times and each time mclust was run to search for components. The size of the shifts was chosen from a Gaussian distribution with the dispersion equal to the velocity distribution of galaxies in the cluster. The number of components found by mclust remained unchanged showing that the component finding was not sensitive to such errors. For details of the analysis of the cluster A2142 with mclust, we refer to Einasto et al. (2012, 2015).

We show the results of the analysis of the substructure in A2142 with mclust in Fig. 4, where we plot the distribution of galaxies in the cluster A2142 in the plane of the sky and in the sky-velocity plane, and show different components in A2142 and its outskirts region. Velocities of galaxies have been calculated from their comoving distances.

In A2142 mclust revealed three components, C1-C3 (Table 1). The distribution of galaxy velocities in the components largely overlap (Fig. 4). The richest of the components, $\mathrm{C} 1$, corresponds to the main cluster with the maximal radius in the sky, $R_{\max }=1.8 \mathrm{~h}^{-1} \mathrm{Mpc}$.

Galaxies from the second component, $\mathrm{C} 2$, plotted in blue in Fig. 4, have velocity distributions similar to the galaxies from the main cluster (C1). As seen in the upper panel of Fig. 4, in the sky plane they are located in the outskirts of the main cluster. To use as much information as possible about the structures in the outskirts region of the cluster, our next step was to compare the components found with mclust with the data from filament catalogue by Tempel et al. (2014a) that was briefly described in Sect. 2.1. This comparison showed that close to the main cluster (our component $\mathrm{C} 1$ ) the filament catalogue lists two short elongated structures. Galaxies from these structures are mostly members of the second component, $\mathrm{C} 2$. These galaxies are denoted as subclusters M1 and M2 in Fig. 4. The filaments very close to the clusters are not well defined, and therefore we refer to these systems as "subclusters". Some galaxies from the first subcluster, M1, are projected inside the virial radius of the main cluster and, according to mclust, belong to the component C1 (see Fig. 4), but following the previous analysis we assign these galaxies to the subcluster M1. Figure 4 shows that in the outskirts of the cluster there are galaxies between subclusters M1 and M2. In what follows, we assign these galaxies to the third subcluster, M3. Below we show that subclusters M2 and M3 have different galaxy content and orientations than M1, which suggests that they are different subclusters.

In the outskirts of the cluster mclust also found a third, very poor component, C3. This component has only eight member galaxies.

Summarising, Fig. 4 shows that the structure of the cluster and its outskirts is rather complex, having several subclusters, revealed using combined results of different structure finding algorithms. We continue the analysis of these structures and galaxy populations in them below.

\footnotetext{
3 http://www.r-project.org
}
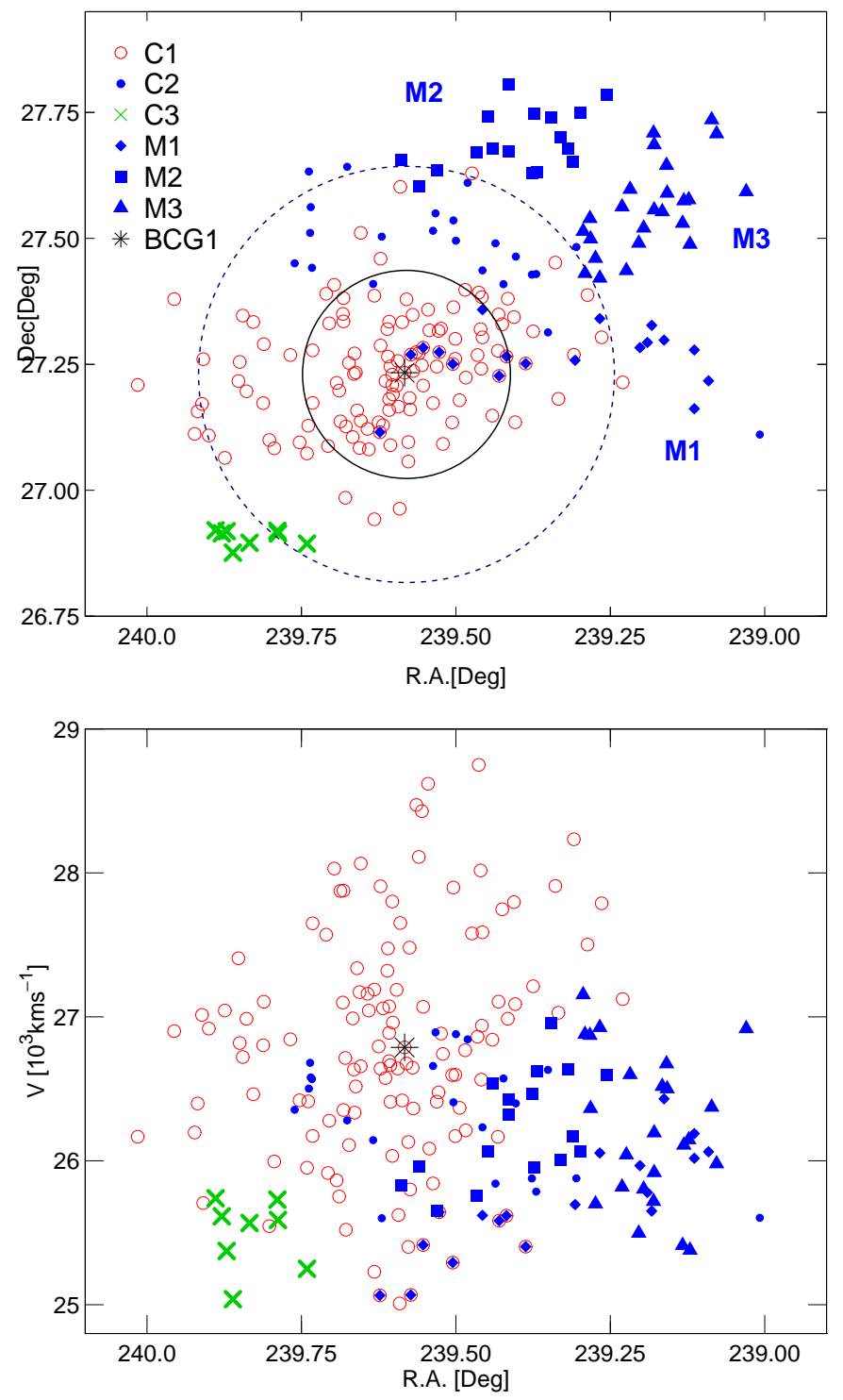

Fig. 4. Distribution of galaxies in the cluster A2142 and its outskirts in the sky plane (upper panel) and in the RA - velocity plane (lower panel) in three components found by mclust. Red symbols correspond to the galaxies in the first component, $\mathrm{C} 1$. Blue symbols show galaxies from the second component, $\mathrm{C} 2$, and green symbols denote the location of galaxies from the third component, $\mathrm{C} 3$. Galaxies from the component C2 are divided into subclusters M1 (diamonds), M2 (squares), M3 (triangles), and dots (those component $\mathrm{C} 2$ galaxies that are not associated with subclusters) as explained in the text. $\mathrm{C} 1$ member galaxies assigned to the subcluster M1 are shown with red circles with blue diamonds (see text). The radius of the small black circle corresponds to the virial radius of the cluster, $R_{\mathrm{vir}}=0.9 \mathrm{~h}^{-1} \mathrm{Mpc}$, and the radius of the large blue circle (dotted line) corresponds to the radius of the component $\mathrm{C} 1$ (main cluster), $R_{\max }=1.8 \mathrm{~h}^{-1} \mathrm{Mpc}$.

In addition to this analysis, previous studies have identified several possible infalling groups in A2142. We show them in Fig. 5 where we plot the distribution of galaxies in the cluster A2142 and its subclusters in the plane of the sky and in the skyvelocity plane and use different colours for galaxies with old and young stellar populations.

In Fig. 5 the location of the two brightest galaxies in the cluster (BCG1 and BCG2) is denoted with stars. The lower panel of Fig. 5 shows that the BCG2 has high velocity with respect to the mean velocity of the cluster (see also Markevitch et al. 2000; Owers et al. 2011). This galaxy may be the brightest 

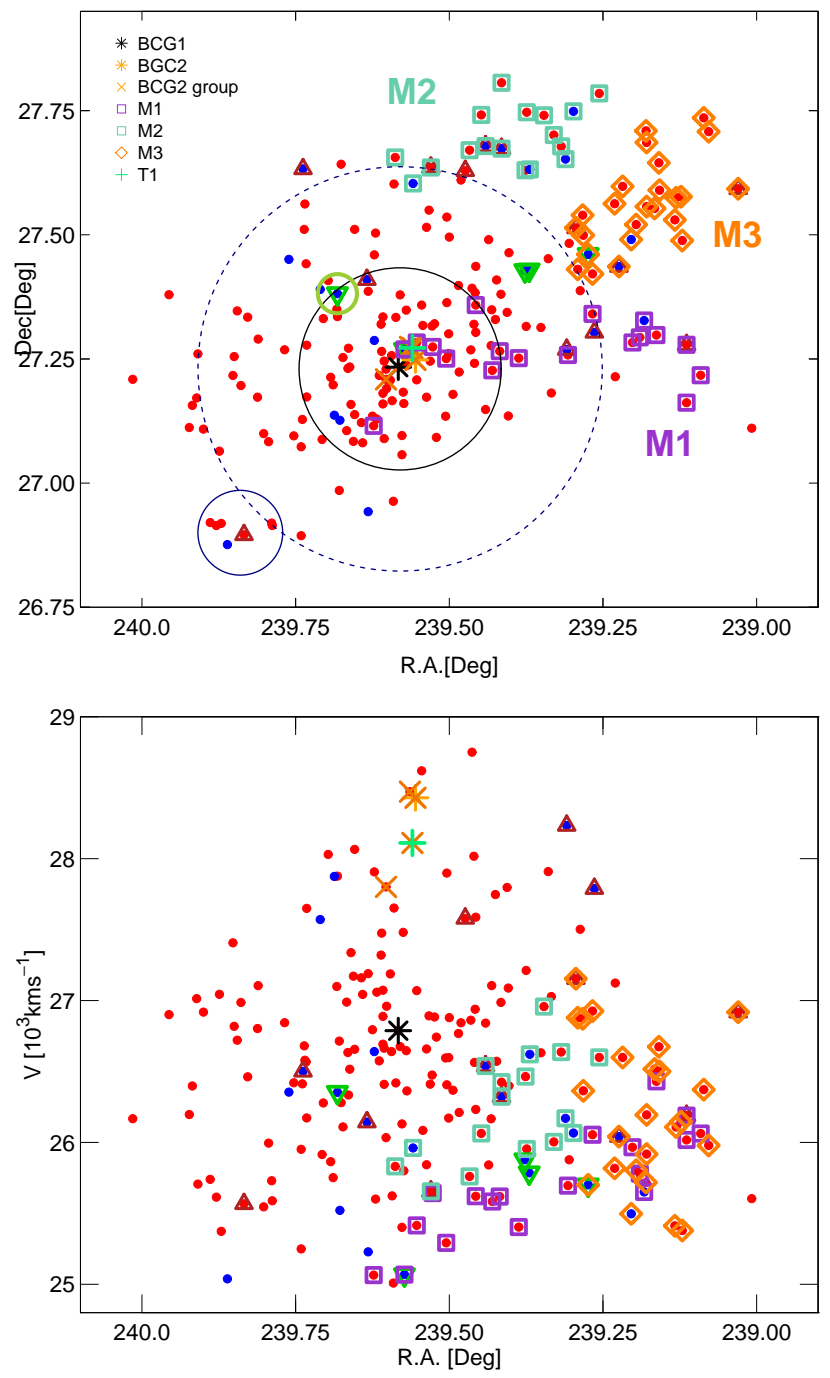

Fig. 5. Distribution of galaxies in the cluster A2142 and its outskirts in the sky plane (upper panel) and in the RA - velocity plane (lower panel). Red filled circles correspond to the galaxies with old stellar populations $\left(D_{n}(4000) \geq 1.55\right)$ and blue filled circles to the galaxies that have young stellar populations with $D_{n}(4000)<1.55$. Green triangles show recently quenched galaxies with $D_{n}(4000) \leq 1.55$ and star formation rate $\log S F R<-0.5$. Red triangles indicate red, high SFR galaxies defined as $g-r \geq 0.7$, and $\log S F R \geq-0.5$ (as in Fig. 3). The black star shows the location of the brightest cluster galaxy (BCG1), and the orange star shows the location of the second BCG (BCG2). The orange crosses show galaxies from a possible BCG2 group. The green cross near the centre denotes a galaxy with a radio tail (T1 in Venturi et al. 2017). The dark violet squares indicate galaxies from subcluster M1, the aquamarine squares indicate galaxies from subcluster M2, and the orange diamonds show galaxies belonging to the third subcluster, M3. The small green circle shows the location of galaxies from infalling group $G_{\mathrm{E}}$ described in Eckert et al. (2014), and the navy circle shows the location of galaxies from the poor group, which corresponds to the third component of the cluster (Einasto et al. 2015). The radius of the black circle corresponds to the virial radius of the cluster, $R_{\mathrm{vir}}=0.9 \mathrm{~h}^{-1} \mathrm{Mpc}$, and the radius of the large blue circle (dotted line) corresponds to the radius of the component $\mathrm{C} 1$ (main cluster), $R_{\max }=1.8 \mathrm{~h}^{-1} \mathrm{Mpc}$.

galaxy of merged group or cluster (Markevitch et al. 2000). Other possible members of the BCG2 group that have very close sky positions and similar velocities as the BCG2 are also indicated. These members correspond to the substructure S1 in Owers et al. (2011). Moreover, Venturi et al. (2017) have de- scribed two long tailed radio sources, named T1 and T2 (see also Govoni et al. 2010). The T1 galaxy is one of the possible BGC2 group members that is mentioned in Markevitch et al. (2000). We indicate this galaxy in Fig. 5 as well.

Eckert et al. (2014, 2017) detected a galaxy group infalling into A2142. Some galaxies from our sample lie at the edge of this group, denoted as $G_{\mathrm{E}}$. We indicate the location of these galaxies in Fig. 5 with a green circle.

Orientations and alignments. We analysed the position angles in the sky plane of the whole cluster A2142 and both BCGs, and compared these with the orientations of the X-ray and radio haloes of the cluster (see Rossetti et al. 2013; Farnsworth et al. 2013; Tchernin et al. 2016; Venturi et al. 2017). We also found the position angle of the supercluster axis determined by the distribution of galaxy groups with at least 10 member galaxies in the supercluster, and we found the position angles of subclusters M1-M3. To determine the position angle of the supercluster, the cluster (including subclusters), and individual subclusters, we approximated their shape with the ellipse and found the position angle of its major axis. We show the direction of the supercluster axis in Fig. 1 (black dashed line) and 6 (black dotted line), where we plot the sky distribution of galaxies in the cluster A2142 and in subclusters. We also plot in Fig. 6 the directions of radio tails of galaxies T1, which coincides with the axis of subcluster M1, and T2 (pointing towards M2) from Venturi et al. (2017), and approximate the contours of the X-ray and radio haloes with ellipse (Venturi et al. 2017).

The position angles of the cluster and supercluster axis approximately coincide, having values $50 \pm 3^{\circ}$ (A2142 cluster) and $63 \pm 1^{\circ}$ (supercluster axis), measured counterclockwise from west (see also Figs. 1 and 5). Component C3 also lies at the supercluster axis. Also the position angles of the visible major axis of both BGCs are close to these angles $\left(\approx 42^{\circ}\right)$. X-ray and radio haloes are aligned along the same axis (Fig. 6 and Venturi et al. 2017).

The directions of tails of radio sources $\mathrm{T} 1$ and $\mathrm{T} 2$ (Venturi et al. 2017) coincide with the directions at which subclusters M1 and M2 are located. In the same time, both galaxies $\mathrm{T} 1$ and $\mathrm{T} 2$ are far from subclusters M1 and M2, as they are located in the centre of the cluster in sky coordinates, at the projected distance approximately $1.8-2 \mathrm{~h}^{-1} \mathrm{Mpc}$ from subclusters. The direction of their tails may be just a coincidence. However, Liu et al. (2016) have mentioned that the presence of the tailed radio galaxy in A2142 may be explained by significant bulk motions in the intercluster medium. Thus the agreement of the directions of galaxy radio tails with substructures suggest the possibility that the infall of subclusters affect the properties of these galaxies.

The subcluster M3 is positioned on the supercluster axis and its position angle is coincident with the supercluster axis (Fig. 6). This agrees with simulations showing that infalling galaxy groups may be elongated along the direction of infall because they are stretched by the cluster tidal field (Vijayaraghavan \& Ricker 2013), and supports the suggestion that we evidence an infall of galaxies along this axis into the cluster.

\subsection{Galaxy populations in the cluster A2142}

Next we analyse galaxy populations in the cluster A2142 and its outskirts structures with the emphasis on their star formation histories. To characterise galaxy populations at a glance, we plot $D_{n}(4000)$ index vs. stellar mass of galaxies for galaxies from various structures determined in the cluster A2142 and its outskirts 


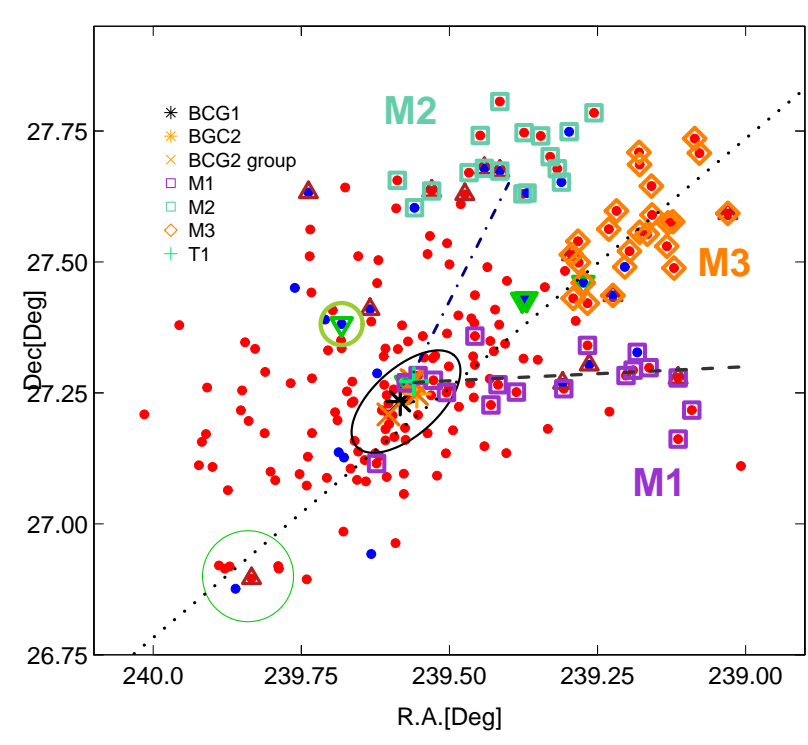

Fig. 6. Distribution of galaxies in the cluster A2142 and its outskirts in the sky plane. The symbols are the same as in Fig. 5. The lines show the direction of supercluster axis (dotted line), subcluster M1 axis (dashed line, this direction coincides with the direction of radio tail of the galaxy $\mathrm{T} 1$, see text), and subcluster M2 direction (dash-dotted line, coincides with the direction of radio tail of the galaxy T2). The ellipse shows approximately the contour of the X-ray halo of the cluster and radio emission at $1.38 \mathrm{GHz}$ (see Venturi et al. 2017, for details).

in upper panels of Fig. 7. In lower panels of these figures, we plot the distributions of each parameter in the main cluster (component $\mathrm{C} 1$ ) and in subclusters taken together (component $\mathrm{C} 2$ ), and separately in three subclusters M1-M3. We do not show error limits in the probability density distributions in lower panels of Fig. 7 since Poisson errors are sensitive to binning the data and may be misleading, especially for bimodal and asymmetrical distributions. We used the Kolmogorov-Smirnov test to find the statistical significance of the differences in $D_{n}(4000)$ indexes for galaxies in the main cluster and in outskirts (C2), using full data (the integral distributions). For details of this approach, we refer to Einasto et al. (2008). We consider that the differences between distributions are highly significant if the $p$ - value (the estimated probability of rejecting the hypothesis that distributions are statistically similar) $p \leq 0.01$.

Figure 7 shows that most galaxies in the main cluster have old stellar populations with no active star formation. In the main cluster $91 \%$ of galaxies have $D_{n}(4000) \geq 1.55$. The median value of the $D_{n}(4000)$ index for the main cluster is $D_{n}(4000)_{\text {med }}=1.9$. In the outskirts of component C2 the percentage of galaxies with $D_{n}(4000) \geq 1.55$ is lower, $82 \%$, and the median value of the $D_{n}(4000)$ index $D_{n}(4000)_{\text {med }}=1.8$ (Fig. 7). The KS test showed that the differences between $D_{n}(4000)$ index values are highly significant $(p<0.01)$.

The lower panels of Fig. 7 suggest that the distribution of $D_{n}(4000)$ indexes for galaxies in the subcluster M1 is similar to that for the main cluster galaxies. In subcluster M3, $D_{n}(4000)_{\text {med }}=1.8$. The lower panels of the figure show that galaxies with the same stellar mass in the main cluster and in the subcluster M3 have different distributions of $D_{n}(4000)$ indexes and therefore different star formation histories. For very old stars this could also be different metallicities, which have an effect on $D_{n}(4000)$ (Kauffmann et al. 2003a). The subcluster M2 (Fig. 7, lower left panel) embeds the largest percentage of star-forming galaxies among subclusters. Here $35 \%$ of galaxies have young
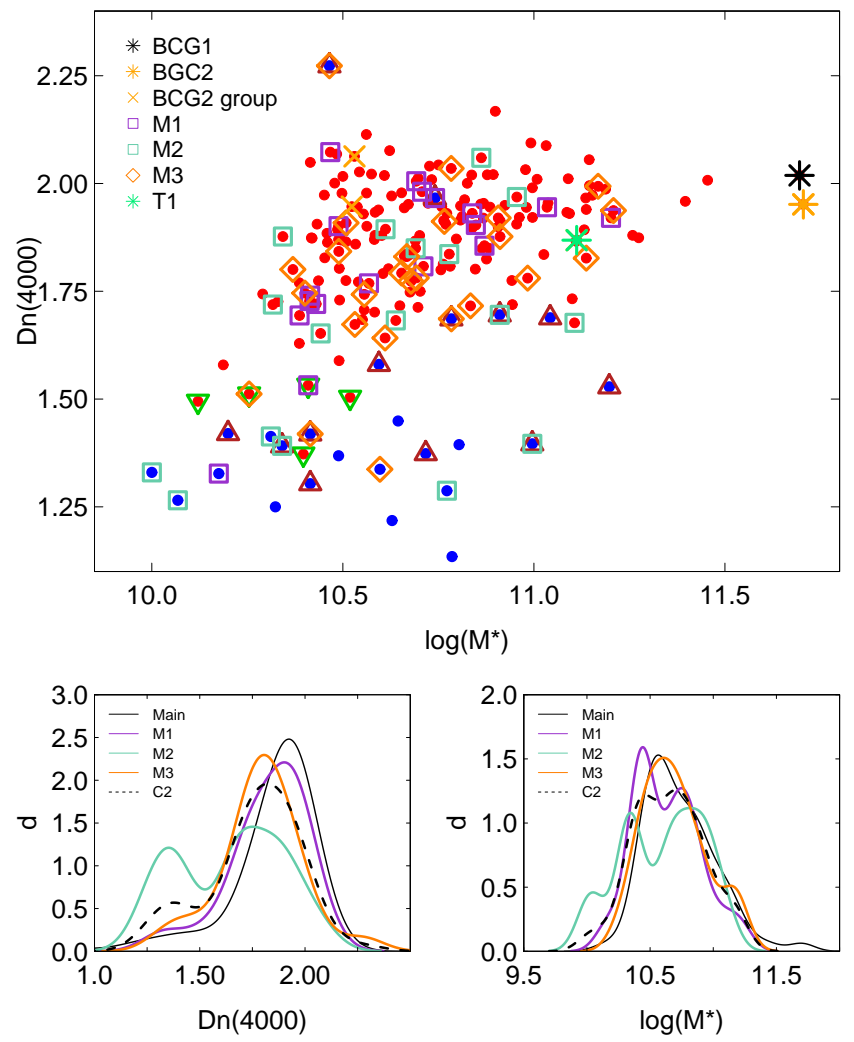

Fig. 7. Upper panel: $D_{n}(4000)$ index vs. stellar mass for the cluster A2142. Red circles indicate low star formation rate galaxies with $\log S F R<-0.5$, and blue circles shows high star formation rate galaxies with $\log S F R \geq-0.5$. Other notations are as in Fig. 5. Lower panels: probability density distributions of $D_{n}(4000)$ (left), and stellar mass (right) of galaxies in the main cluster (black solid line), in the outskirts component $\mathrm{C} 2$ (subclusters M1-M3 taken together, black dashed line), and in subclusters M1-M3 (M1 - violet line, M2 - aquamarine line, and M3 - orange line).

stellar populations and $D_{n}(4000)_{\text {med }}=1.7$. This subcluster has the lowest stellar masses of galaxies (Fig. 7, lower right panel). According to Haines et al. (2017b), lower stellar mass galaxies also have lower $D_{n}(4000)$ values. The different stellar mass distribution probably can explain the different $D_{n}(4000)$ distribution. However, the number of galaxies in subclusters is rather small. This means that the differences mentioned here have low statistical significance and can only be taken as suggestions.

Galaxies from the possible BCG2 group (see Fig. 5) have as old stellar populations as the main cluster galaxies, where the median value of the $D_{n}(4000)$ index $D_{n}(4000)=1.9$. It is interesting to note that the galaxy identified with the radio source T1 from Venturi et al. (2017) has the lowest $D_{n}(4000)$ index value among the galaxies from the possible BCG2 group. The stellar age estimates of BGCs and T1 galaxy differ even more; they are 12.0 and $6.5 \mathrm{Gyr}$, respectively. This gives additional support to the suggestion that the infall of galaxies from M1 subcluster affect the properties of this galaxy.

We defined recently quenched galaxies as those with $D_{n}(4000) \leq 1.55$ and star formation rate $\log S F R<-0.5$ (five galaxies). Red, high SFR galaxies are defined as galaxies with $(g-r)_{0} \geq 0.7$, and $\log S F R \geq-0.5$ (13 galaxies).

Galaxies with low star formation rates and young stellar populations (recently quenched galaxies with $D_{n}(4000) \leq 1.55$ and star formation rate $\log S F R<-0.5)$ reside mostly in the transition region of Fig. 7 and have intermediate $D_{n}(4000)$ index 


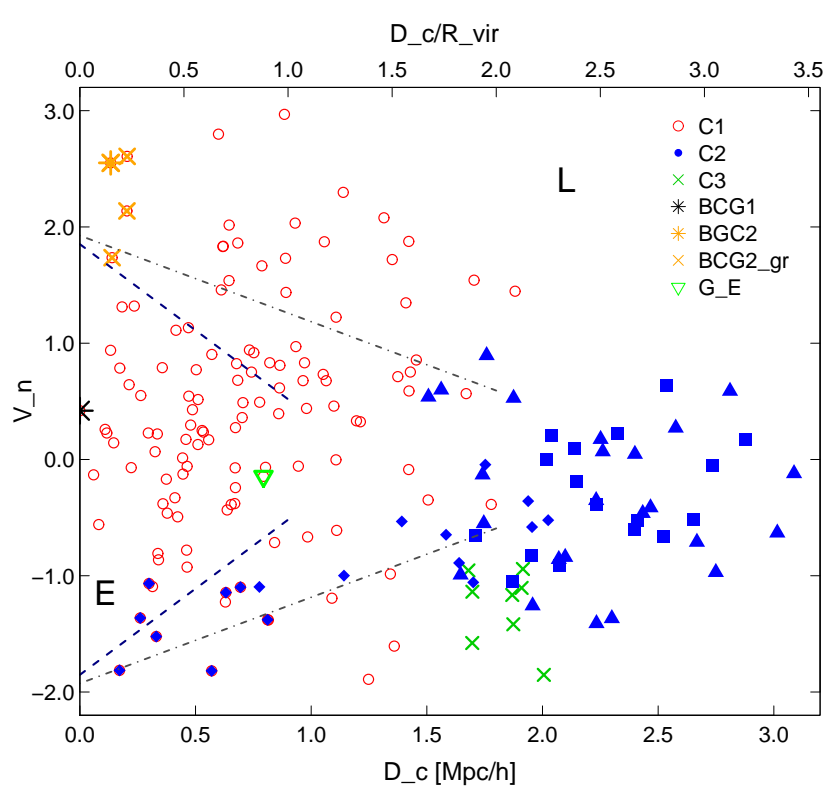

Fig. 8. Normalised velocity of galaxies with respect to the cluster mean velocity vs. projected clustercentric distance for the cluster A2142, its outskirts subclusters, and infalling groups (see text). Lines separate approximately the early and late infall galaxies with increasing time of infall; two different boundaries separate early and late infall time galaxies. The earliest infall galaxies with infall time $t_{\text {inf }}>1 \mathrm{Gyr}$ lie between the black dashed lines, calculated using $R_{\mathrm{vir}}$, and late or ongoing infall galaxies with infall time $t_{\text {inf }}<1 \mathrm{Gyr}$ lie above and below the lines, and on the right side of the lines (see Oman et al. 2013; Agulli et al. 2017, for details). The grey dash-dotted lines are calculated using $R_{\max }$ (see text). Notations of components C1-C3 and M1-M3 are the same as in Fig. 4. Notations of the BCGs and possible infalling groups are given in legend; E indicates the early infall region and L denotes the late infall region.

values. Most galaxies, which are red in colour and have high star formation rates with $(g-r)_{0} \geq 0.7$, and $\log S F R \geq-0.5$, also reside here. Three recently quenched galaxies are located in the subcluster M3, two of which belong to a close pair of galaxies. One recently quenched galaxy is located in the subcluster M1, and one is located at the edge of the infalling group $G_{\mathrm{E}}$ (Fig. 5).

\subsection{Phase space analysis of the cluster A2142 and its outskirts}

We employ PPS diagram of A2142 to separate regions of different dynamical states and infall times and to analyse galaxy populations in these regions. Corresponding PPS diagrams for the cluster A2142 are shown in Figs. 8 and 9. The PPS diagrams show line-of-sight velocities of galaxies with respect to the cluster mean velocity vs. projected clustercentric distance. The differences between galaxy velocities and the cluster mean velocity are normalised by the cluster velocity dispersion, $v_{\mathrm{n}}=\left(v-v_{\text {mean }}\right) / \sigma_{\mathrm{cl}}$. Several recent studies have shown that in the phase space diagram galaxies with different accretion histories populate different areas of the diagram (Oman et al. 2013; Muzzin et al. 2014; Haines et al. 2015; Jaffé et al. 2015; Agulli et al. 2017; Paccagnella et al. 2017; Yoon et al. 2017; Rhee et al. 2017). Galaxies with early infall times are located at small clustercentric distances in the central part of the figure (virialised region), while recently infallen or still infalling galaxies lie at large clustercentric distances and/or have high velocities with respect of the cluster mean velocity (nonvirialised region).

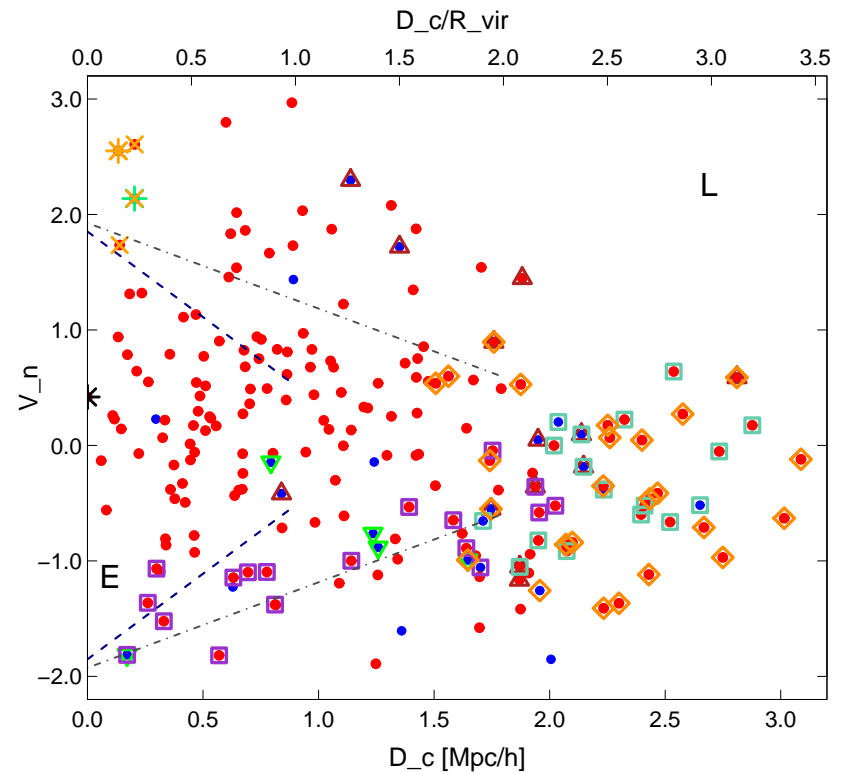

Fig. 9. Normalised velocity of galaxies with respect of the cluster mean velocity vs. projected clustercentric distance for the cluster A2142 and its outskirts. Lines separate approximately the early and late infall galaxies with increasing time of infall; two different boundaries separate early and late infall time galaxies. Earliest infall galaxies with infall time $t_{\text {inf }}>1 \mathrm{Gyr}$ lie between black dashed lines, calculated using $R_{\mathrm{vir}}$, and late or ongoing infall galaxies with infall time $t_{\text {inf }}<1$ Gyr lie above and below the lines, and on the right side of the lines (see Oman et al. 2013; Agulli et al. 2017, for details). Grey dash-dotted lines are calculated using $R_{\max }$. Notations are the same as in Fig. 5; E indicates the early infall region and $\mathrm{L}$ indicates the late infall region.

Early and late infall time regions can be approximately separated with a line corresponding to the infall time of the cluster member galaxies (Oman et al. 2013) as follows:

$\left|\frac{v-v_{\text {mean }}}{\sigma_{\mathrm{cl}}}\right|=-\frac{4}{3} \frac{D_{\mathrm{c}}}{R_{\mathrm{vir}}}+2$,

where $v$ are the velocities of the galaxies, $D_{\mathrm{c}}$ is the projected clustercentric distance, and $R_{\mathrm{vir}}$ is the cluster virial radius. Oman et al. (2013) showed that in simulations a large percentage of galaxies on the left of the line at small clustercentric radii form an early infall (virialised) population with $\tau_{\text {inf }}>1 \mathrm{Gyr}$. Galaxies on the right of this line at large clustercentric radii fell into the cluster during the last $1 \mathrm{Gyr}$. These galaxies form late or ongoing infall populations with an infall time $\tau_{\text {inf }}<1 \mathrm{Gyr}$. Simulations show that galaxies with late infall time may populate areas of PPS diagram at all projected radii and velocities, which complicates the interpretation of the PPS diagram (Haines et al. 2015).

In Figs. 8 and 9 we use two lines to separate different regions of A2142 in the PPS diagram. Both are calculated with Eq. (3). The dashed line corresponds to the virial radius as defined in Eq. (1); it separates the inner, virialised region of the cluster. The dot-dashed line corresponds to maximal radius of the components $\mathrm{C} 1, R_{\max }$. This line indicates the outer region of the main cluster where subclusters enter the cluster. We plot both set of lines to see how sensitive the PPS analysis is to the definition of the virialised region.

Figure 8 presents the PPS diagram for substructures C1$\mathrm{C} 3$ and subclusters of the component $\mathrm{C} 2$. We also show here galaxies from the BCG2 group and possible $G_{\mathrm{E}}$ group member. This figure shows that almost all galaxies at clustercentric distances approximately $D_{\mathrm{c}}<0.6 \mathrm{~h}^{-1} \mathrm{Mpc}$ belong to the early infall 

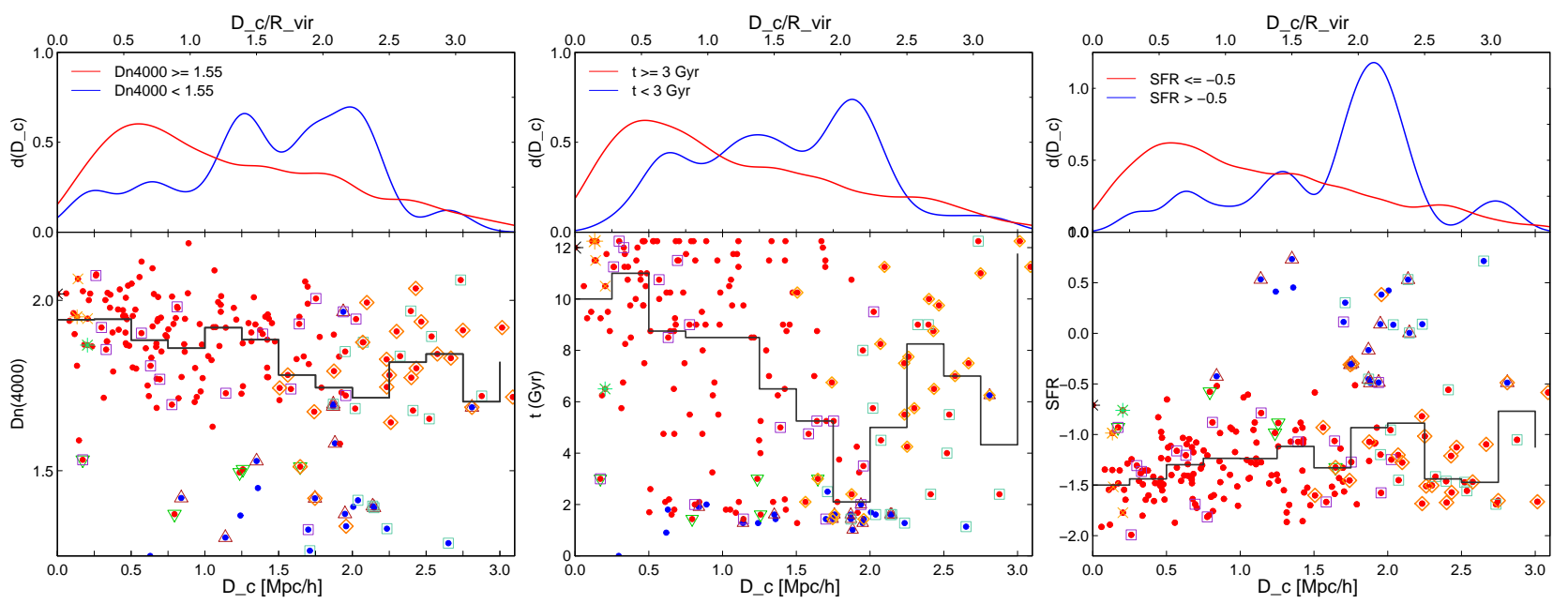

Fig. 10. $D_{n}(4000)$ index (left panel), ages of stellar populations (middle panel), and star formation rates (right panel) for galaxies in A2142 vs. clustercentric distance. The upper panels show the distribution of clustercentric distances for galaxies with young and old stellar populations, and passive and actively star-forming galaxies (see legends in the panels). In the lower panels, the black line shows median values of parameters. Notations are as in Fig. 5, upper panel.

(virialised) region and are members of the main component $\mathrm{C} 1$. Galaxies from the BCG2 group in the cluster centre in sky coordinates have very high velocities with respect to the cluster mean velocity (noted also in Markevitch et al. 2000). These galaxies may have already passed cluster centre (Oman et al. 2013). The galaxy at the edge of the group $G_{\mathrm{E}}$ lies at the clustercentric distance approximately $0.7 \mathrm{~h}^{-1} \mathrm{Mpc}$, at the edge of the virialised region.

The members of subclusters M1-M3 and component C3 lie in the late infall region of the PPS diagram. These members may still be infalling. Also, some subcluster M1 galaxies, although near the cluster centre according to the projected distance, lie in the region between two lines and may still be infalling. Owers et al. (2011) detected several substructures in the direction of the subcluster M1 (their S5-S7). Infall of subcluster M1 galaxies in this region may be the cause of the radio ridge described in Venturi et al. (2017).

According to the position in the PPS diagram, the component C3 may represent another poor galaxy group infalling to the main cluster along the supercluster axis.

Figure 8 also shows that some galaxies from the main cluster component, $\mathrm{C} 1$, outside of the early infall region at clustercentric distances, $D_{\mathrm{c}}>0.7 \mathrm{~h}^{-1} \mathrm{Mpc}$, have high positive velocities with respect to the cluster centre. Simulations suggest that such galaxies may belong to a population yet to be accreted (see e.g. Fig. 13 in Haines et al. 2015).

In Fig. 9 most galaxies of the main cluster within the early infall region, including galaxies from the BCG2 group, have old stellar populations $\left(D_{n}(4000) \geq 1.55\right)$. One recently quenched galaxy and one red star-forming galaxy lie at the edge of the infalling group $G_{\mathrm{E}}$. Their properties could be affected by the infalling group.

In Fig. 9, star-forming (including red star-forming) and recently quenched galaxies reside mostly in the region at approximately $1.5-2.0 \mathrm{~h}^{-1} \mathrm{Mpc}$ from the cluster centre at the outskirts of $\mathrm{C} 1$ connected with subclusters M2 and M3, which are probably infalling. Galaxies from the poorest cluster component determined by mclust (one red and one blue star-forming galaxy among these members) are also located here. At clustercentric distances larger than $2 \mathrm{~h}^{-1} \mathrm{Mpc}$, subclusters $\mathrm{M} 1$ and $\mathrm{M} 3$ are populated mostly by passive, non-star-forming galaxies; star- forming galaxies belong mostly to the subcluster M2. We analyse how the properties of galaxies in A2142 and its outskirts change with the clustercentric distance in more detail in next subsection.

\subsection{Galaxy populations in A2142 vs. projected clustercentric distance}

Next we study how the galaxy populations in the cluster and subclusters change with the clustercentric distance with a focus on star formation indicators. We calculate the distributions of clustercentric distances for galaxies with various star formation properties. We use parameter limits as given before. Quiescent galaxies with old stellar populations have $D_{n}(4000) \geq 1.55$, and star-forming galaxies with young stellar populations have $D_{n}(4000)<1.55$. Stellar age limit $t=3$ Gyr separates galaxies with old and young stellar populations, galaxies with old stellar populations have $t \geq 3 \mathrm{Gyr}$, and those with young stellar populations have $t<3$ Gyr. Star formation rate $\log S F R \leq-0.5$ corresponds to quiescent galaxies, and $\log S F R \geq-0.5$ to starforming galaxies. In upper panels of Fig. 10, we show the distributions of clustercentric distances for galaxies from these populations. Distributions are normalised, so that each integrates to 1 . In lower panels of this figure, we show the $D_{n}(4000)$ index, age, and star formation rates for all galaxies from our sample. Additionally, in Fig. 11 we show the stellar velocity dispersions and concentration index (the ratio of the Petrosian radii $R_{50} / R_{90}$ ) for galaxies in $\mathrm{A} 2142$ vs. clustercentric distance. We also plot median values of the parameters for the full cluster. In the upper panels, distributions are calculated for complete sample, excluding faint galaxies below the completeness limit, $M_{r}=-19.6+5 \log _{10} h$. The values of parameters are known for all galaxies (except one galaxy).

The upper panels of Fig. 10 show that the distribution of passive, old galaxies without ongoing star formation has maximum at clustercentric distances approximately $0.5 \mathrm{~h}^{-1} \mathrm{Mpc}$ in the central, most virialised part of the main cluster. At higher clustercentric distances the percentage of passive galaxies decreases and star-forming galaxies become more frequent. All panels of Fig. 10 show that the maximum in the distance distribution of star-forming galaxies lie approximately at the distance 

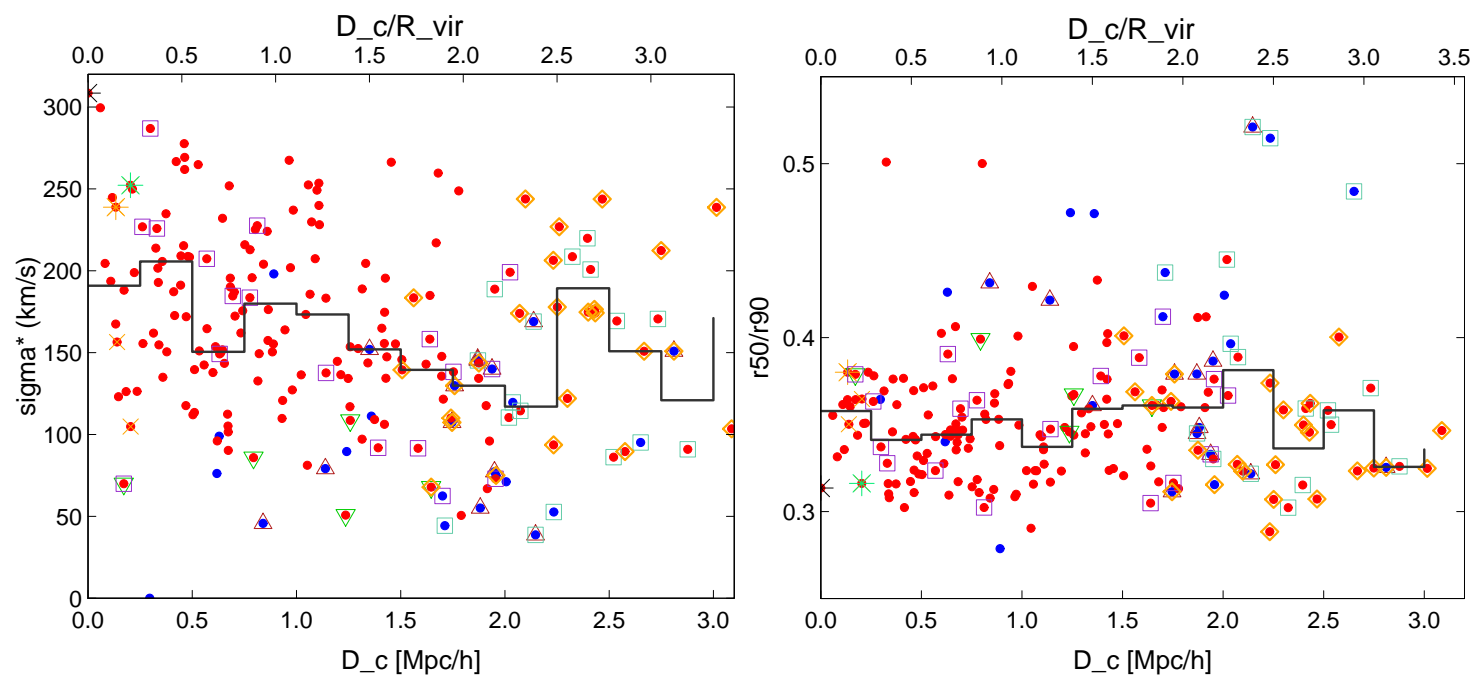

Fig. 11. Stellar velocity dispersions (left panel) and concentration index (the ratio of the Petrosian radii $R_{50} / R_{90}$, right panel) for galaxies in $\mathrm{A} 2142$ vs. clustercentric distance. The black line shows the median values of parameters. Notations are as in Fig. 5, upper panel.

of $1.8 \mathrm{~h}^{-1} \mathrm{Mpc}$. The KS test shows that the differences between clustercentric distance distributions of passive and star-forming galaxies have very high statistical significance (with $p<0.01$ ) for all three parameters.

The lower panels of Fig. 10 show that median value of the $D_{n}(4000)$ index for galaxies in the cluster centre at the clustercentric distances $D_{\mathrm{c}}<0.5 \mathrm{~h}^{-1} \mathrm{Mpc}$ is $D_{n}(4000)=1.95$ and the median age of stellar populations is $t \approx 10-11 \mathrm{Gyr}$. The galaxy T1 from a possible BCG2 group has the lowest age among the BCG2 group galaxies and the highest star formation rate.

At the clustercentric distances $D_{\mathrm{c}}>0.5 \mathrm{~h}^{-1} \mathrm{Mpc}$, median values of galaxy parameters start to change. At the clustercentric distance interval $1.75<D_{\mathrm{c}}<2.25 \mathrm{~h}^{-1} \mathrm{Mpc}$, at the boundaries of the main cluster where subclusters fall into the cluster almost all galaxy parameters change rapidly. The age of stellar populations of galaxies have median value of 2 Gyr only, and the median value of the $D_{n}(4000)$ index is $D_{n}(4000)=1.7$. Stellar velocity dispersions of galaxies have the lowest median values and galaxies become less compact. The recently quenched galaxies and most star-forming galaxies in subclusters M1, M2, and M3 lie in this distance interval. The KS test shows that the differences between the parameter values in the centre of the cluster (at clustercentric distances $D_{\mathrm{c}}>0.5 \mathrm{~h}^{-1} \mathrm{Mpc}, 42$ galaxies from the complete sample) and at the virial radius (in the clustercentric distance interval $1.75<D_{\mathrm{c}}<2.25 \mathrm{~h}^{-1} \mathrm{Mpc}, 33$ galaxies) are highly significant with $p<0.01$.

Higher stellar velocity dispersions in the central part of the cluster, in the region of early infall in the phase space diagram (Fig. 9) are in agreement with the results that show that galaxies with old stellar populations have higher stellar velocity dispersion values than galaxies with young stellar populations (Shankar et al. 2009; Bezanson et al. 2012).

As the clustercentric distance increases $\left(D_{\mathrm{c}}>\right.$ $\left.2.25 \mathrm{~h}^{-1} \mathrm{Mpc}\right)$, stellar ages of galaxies increase together with the increase of their $D_{n}(4000)$ index and stellar velocity dispersion. The median age of stellar populations of galaxies in the distance interval of $2.25<D_{\mathrm{c}}<2.5 \mathrm{~h}^{-1} \mathrm{Mpc}$ is $8 \mathrm{Gyr}$. The concentration index of galaxies increases and then decreases again. In this distance interval star-forming galaxies belong to subcluster M2.

Together these figures suggest that in a narrow clustercentric distance interval, the star formation of galaxies in subclusters is enhanced at the boundaries of the cluster where subclusters enter the main cluster and is accompanied by structural changes of galaxies and changes in other properties of galaxies. This suggest that the infall of galaxies into the cluster enhances their star formation, also affecting their other properties.

\section{Discussion}

\subsection{Properties of galaxies at various clustercentric distances}

We found the change in galaxy populations in the cluster A2142 at the clustercentric distances of about $1.6-1.8 \mathrm{~h}^{-1} \mathrm{Mpc}$, which is probably evidence of a merger-induced star formation activity in the region where subclusters fall into the main cluster. This has also been found in other studies. For example, Porter et al. (2008) and Mahajan et al. (2012) detected enhanced star formation in galaxies in filaments entering into galaxy clusters. Deshev et al. (2017) have detected a high percentage of star-forming galaxies in the cluster A520 in infalling groups at $R_{200}$ and higher clustercentric distances. Baxter et al. (2017) have shown that colours of galaxies from the SDSS change at the halo boundaries. Park \& Hwang (2009) found that at the characteristic scale approximately $1-3 R_{200}$ (for bright galaxies this radius is smaller than for faint galaxies) the properties of galaxies start to depend on the clustercentric radius at a fixed nearest neighbour environment. In the same time, some other studies detected smooth changes in galaxy populations in clusters with the increase of clustercentric distance (Haines et al. 2015; Paccagnella et al. 2016). These studies propose that, for example, interactions of galaxies with intracluster medium causes a gradual shutdown of star formation. Our results in Sect. 3.4 show both the slow changes in galaxy properties as the clustercentric distance increases, and a rapid change in galaxy properties at the boundaries of the cluster. This may be related to accretion shocks as gas falls into clusters along the filaments around clusters (Molnar et al. 2009).

\subsection{Central region of A2142: multiwavelength view}

Earlier studies of the central region of A2142 (see references in Introduction) show that the central part of the cluster have a very complicated structure, with many substructures, showing signatures of several mergers. We described some of these 
merging structures and their galaxy populations in this paper. Among these structures are infalling groups and subclusters. One recently quenched galaxy is found at the edge of infalling group $G_{\mathrm{E}}$, described in Eckert et al. $(2014,2017)$. Stellar populations of galaxies from the possible BCG2 group are very old. Infall of subcluster M1 galaxies may be the cause of the radio ridge described in Venturi et al. (2017). Also, Venturi et al. (2017) have described two tailed radio galaxies in the central region of the cluster (T1 and T2). The directions of the tails coincide with the direction at which subclusters M1 and M2 are located and supports the possibility that these subclusters are infalling and affecting galaxy properties in the cluster.

These studies have also shown that in this cluster the distribution of galaxies follow that of intracluster medium. No signatures of the dark matter offset has been found as in some other rich merging galaxy clusters such as in the cluster A520 (examples of some such galaxy clusters were given recently in Wittman et al. 2017). This can be explained by different timescales of mergers. For A520 the time since merger has been estimated to be less than 0.5 Gyr (Mahdavi et al. 2007; Girardi et al. 2008; Deshev et al. 2017), while for A2142 we propose that the time since major merger is larger and may be of the order of $4 \mathrm{Gyr}$ (see below).

\subsection{Clusters, superclusters, and the cosmic web}

In this study we showed that the cluster A2142, infalling subcluster M3, and one infalling group are aligned along the cluster and supercluster axis (the cluster and supercluster alignments were also discussed in Einasto et al. 2015). The radio and X-ray haloes are also aligned with the supercluster axis (Venturi et al. 2017, and Fig. 6).

The correlated orientations of galaxies, galaxy clusters, and superclusters were noted already in the early studies of the cosmic web. Jõeveer et al. (1978) showed that in the Perseus-Pisces supercluster the main galaxies of the clusters are directed along the chain that connects the Perseus-Pisces supercluster with other nearby superclusters. Practically all clusters in the main chain of the Perseus-Pisces supercluster are elongated along the main ridge of the chain. Jõeveer et al. (1978) concluded that a close physical link exists between cluster main galaxies and their environment, which hints at a common origin and evolution of galaxies and galaxy clusters in the cosmic web. Later many studies have confirmed the presence of alignments of galaxies (especially the brightest cluster galaxies) in groups, clusters, superclusters, and filaments (West \& Blakeslee 2000; Plionis \& Basilakos 2002; Hopkins et al. 2005; Paz et al. 2011; Tempel et al. 2015; Tempel \& Tamm 2015; McIntosh et al. 2016; Hirv et al. 2017; Foëx et al. 2017; West et al. 2017, and references therein). The alignment signal may be a result of how galaxies and galaxy groups fall into clusters along preferred directions - the large-scale filamentary structures within which they are embedded.

Lietzen et al. (2012) and Einasto et al. (2014) compared the percentage of red galaxies in galaxy groups and clusters of similar richness in superclusters and in the field. These authors found that groups in superclusters have higher percentage of red galaxies than groups in lower density environment and that the percentage of red galaxies is the highest in groups and clusters in the high-density cores of rich superclusters.

Our findings about the galaxy content of the main cluster A2142 and subclusters M1 and M3 agree with the result by Lietzen et al. (2012) and Einasto et al. (2014). Galaxies in the subcluster M1 have star formation properties closer to those in the main cluster than in other subclusters. These galaxies lie in the outer region of the cluster with later infall times than galaxies in the central, early infall region. The percentage of star-forming galaxies in the subcluster M2 is higher than in M1 and M3; it is higher than typical for unrelaxed clusters, especially in supercluster cores, $f_{S F} \approx 0.2$ (Cohen et al. 2014, 2017). Also, Luparello et al. (2013) found that galaxy populations in groups residing in superclusters are older than those in groups elsewhere. On the other hand, Einasto et al. (2010) studied the substructure and galaxy content of the richest galaxy clusters from the SGW. These authors used mclust to determine different components in clusters. The analysis of the galaxy content of individual components in clusters showed that in several clusters some components were populated mainly by elliptical galaxies and other components by spiral galaxies, which is in agreement with what we found for the cluster A2142 and subclusters M1M3. The high percentage of star-forming galaxies in the subcluster M2 may be due to anisotropic infall of galaxies such that, in this subcluster, galaxies are affected by the infall up to higher distances from the cluster than in other subclusters.

Einasto et al. (2014) analysed the structure and galaxy content of galaxy superclusters from the SDSS and found that galaxy clusters in superclusters with a more complicated inner structure (so-called superclusters of spider morphology) have a higher percentage of blue star-forming galaxies than galaxy clusters in superclusters with simpler inner structures in which galaxy groups and clusters are connected by a small number of galaxy filaments (filament-type morphology). The A2142 supercluster is of filament morphology and the very high percentage of passive, red galaxies in its main cluster agrees with the finding by Einasto et al. (2014). In the same time we showed that the percentage of star-forming galaxies increases in the infall region of the cluster. This may provide a hint as to why the percentage of star-forming galaxies is high in superclusters in which galaxy clusters are connected by a large number of filaments (spidertype morphology). A large number of filaments increases the possibility of mergers and increases star formation in galaxies.

Costa-Duarte et al. (2013) did not find a difference between stellar populations of galaxies in superclusters classified by their overall shape as filaments and pancakes. In agreement with this result, Einasto et al. (2014) found that superclusters with different overall shapes have similar galaxy populations.

Individual rich superclusters have different inner structures and galaxy and group contents (Einasto et al. 2014). For example, Krause et al. (2013) found that in the Ursa Major supercluster groups with Gaussian galaxy velocity distributions reside in higher density environments than groups with nonGaussian galaxy velocity distributions and have higher densities of red galaxies. The Ursa Major supercluster is classified as having multibranching filament type morphology in Einasto et al. (2011). Ursa Major has several rich clusters, while SCl A2142 is dominated by one very rich cluster. However, the velocity distributions of galaxies in different components in the cluster A2142 overlap, and using velocity data only, it could be classified as Gaussian (Einasto et al. 2012). This cluster resides in the highdensity core of the supercluster and has high percentage of red galaxies, which agrees with the Krause et al. (2013) findings for the Ursa Major supercluster.

Johnston-Hollitt et al. (2008) found an excess of blue starforming galaxies aligned along the axis connecting the cluster A3158 to the cluster A3125/A3128 complex in the core region of the Horologium-Reticulum supercluster. These authors concluded that this is evidence of merger-induced star formation in these galaxies. 


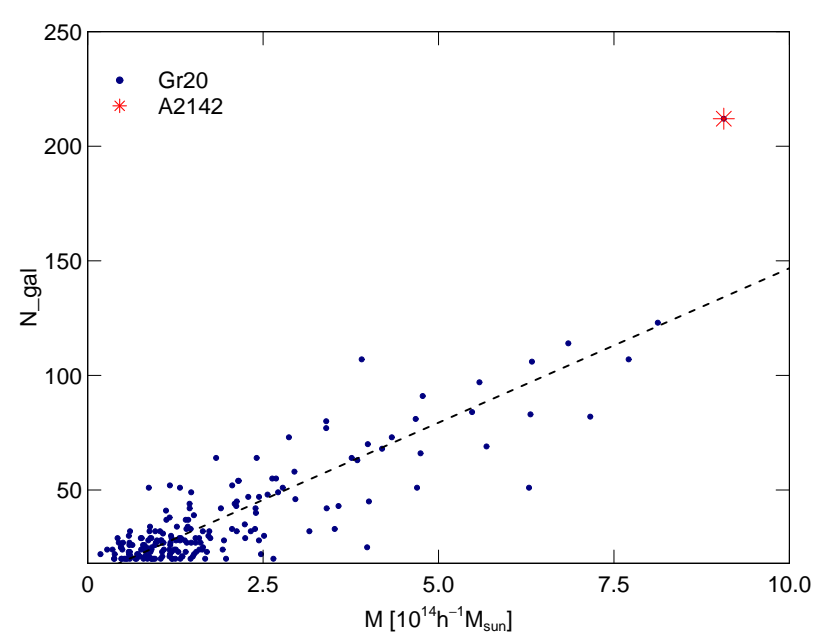

Fig. 12. Masses of galaxy groups with at least 20 member galaxies (dark blue symbols) vs. the group richness in the distance interval 225-280 $\mathrm{h}^{-1}$ Mpc from the group cataloque by Tempel et al. (2014b). The red star denotes the cluster A2142.

Superclusters obtained their group and galaxy populations during the formation and evolution of the cosmic web. The observational results show that the variety of galaxy and group properties in individual superclusters is large. This shows a need to continue the studies of galaxy and group populations in superclusters to understand better their formation and evolution.

\subsection{Comparison with other galaxy groups and clusters}

Now we briefly compare A2142 with other rich groups and clusters from the Tempel et al. (2014b) group catalogue. To avoid strong distance-dependent selection effects, we only used data of groups with at least 20 member galaxies from a narrow distance interval 225-280 $\mathrm{h}^{-1} \mathrm{Mpc}$. Many rich superclusters including the SGW are located at these distances (see also Einasto et al. 2014 , for details about galaxy superclusters from this distance interval).

Figure 12 presents the mass - richness relation for galaxy groups. This figure shows that A2142 is much richer than other groups and clusters in this distance interval. For example, A2142 is twice as rich as the richest galaxy clusters in the SGW, which is a rich supercluster complex at approximately the same distance as SCl A2142 (Einasto et al. 2010, and references therein).

Einasto et al. (2010) studied substructure and galaxy populations of the 10 richest galaxy clusters in the SGW superclusters. These clusters show the presence of substructure, signs of mergers, and perhaps rotation, which suggest that the richest clusters in the SGW are not yet virialised. The mean values of the colour index of galaxies in these clusters, $(g-r)_{\text {mean }} \approx 0.75$, are lower than we found for the cluster A2142 and its substructures in this paper, $(g-r)_{\text {med }} \approx 0.9$. Einasto et al. (2010) also found that the percentage of red galaxies in various SGW superclusters increases with supercluster richness and is higher in clusters that lie in the high-density cores of the SGW superclusters. The percentage of red galaxies is the highest in the clusters A1650 and $\mathrm{A} 1750$ in the cores of the richest SGW supercluster, $f_{\text {red }} \approx 0.9$, which is the same as in the main cluster in this study. The masses of the richest clusters in the high-density cores of the SGW are approximately $M_{\mathrm{cl}} \approx 10^{15} \mathrm{~h}^{-1} M_{\odot}$, that is similar to the mass of the cluster A2142 (Table 1 and Einasto et al. 2016).

Next we compared galaxy populations in A2142 and in other groups and clusters with at least 20 member galaxies in the same distance interval as before, $225-280 \mathrm{~h}^{-1} \mathrm{Mpc}$. In the comparison we only used galaxies brighter than $M_{r}=-19.6+5 \log _{10} h$, which is the completeness limit of A2142. In Fig. 13 we plot distributions of stellar masses, star formation rates, and $(g-r)_{0}$ colours of galaxies in groups with at least 20 member galaxies, and separately in rich clusters with at least 100 member galaxies.

This figure shows that the galaxies in A2142 have higher stellar masses than galaxies of the same absolute magnitude limit in other rich groups and clusters. Also, they have lower star formation rates and redder colours, although, as we mentioned above, the percentage of red galaxies in A2142 and in some rich clusters from the SGW is the same. The KS test shows that the differences between galaxy parameters in A2142 and other groups and clusters are statistically significant at high levels ( $p$ value $p<0.01$ for all parameters).

Therefore, A2142 not only has a much larger number of luminous galaxies than other groups and clusters in this distance interval, the galaxies in A2142 are different, on average, from galaxies in other groups and clusters. They are redder and have higher stellar masses and lower star formation rates. Moreover, Munari et al. (2016) showed that the abundance of member galaxies in haloes of virial masses comparable to that of A2142 from numerical simulations is significantly smaller than in $\mathrm{A} 2142$.

\subsection{Formation of $A 2142$}

The cluster A2142 is an unusually rich cluster in the collapsing core of the supercluster, elongated along the supercluster axis together with the subcluster M3 and component C3. Numerical simulations show that high-density cores of superclusters are the locations where positive sections of density perturbations combine and clusters form via merging and accretion of galaxy groups and clusters (Bond et al. 1996; Suhhonenko et al. 2011; Kravtsov \& Borgani 2012, and references therein). The cluster A2142 is the only rich cluster in the supercluster. We may assume that this cluster has been formed through merging and accretion of other groups and clusters in the supercluster core region. This assumption is supported by the finding that the core region of the A2142 supercluster with radius of about $6-8 \mathrm{~h}^{-1} \mathrm{Mpc}$ has reached turnaround and is collapsing (Einasto et al. 2015; Gramann et al. 2015). In an expanding Universe turnaround radius is a radius at which matter stops expanding with the Universe and starts collapsing. We may expect that galaxy systems surrounding the A2142 cluster up to the turnaround radius in the supercluster core are infalling, which is in agreement with the results of this paper. Along the supercluster axis, the accretion of groups may be enhanced owing to high overall density and this may make the cluster unusually rich.

The mass of $\mathrm{A} 2142$ is of the order of $10^{15} \mathrm{~h}^{-1} M_{\odot}$ (Munari et al. 2014; Einasto et al. 2015). Kim et al. (2015) analysed the evolution of halo mass along the major descendant trees in Horizon Run 4 simulations and showed that massive halos with mass of the order of $10^{15} \mathrm{~h}^{-1} M_{\odot}$ have formed relatively recently. On average, halos with a current mass of $10^{15} \mathrm{~h}^{-1} M_{\odot}$ have their half mass $\left(0.5 \times 10^{15} \mathrm{~h}^{-1} M_{\odot}\right)$ at $z=0.5$ (look-back time approximately 4 Gyr, see also Haines et al. 2017a, and references therein).

Ongoing and recent accretion of groups and subclusters increase the mass of the cluster. Next we give mass estimates of infalling groups and subclusters discussed in this study.

$B C G 2$ group. The second brightest galaxy in the cluster (BCG2) with high peculiar velocity and old stellar populations with $D_{n}(4000)_{\text {med }}=1.9$ and $(g-r)_{0}=0.92$ may be the 
M. Einasto et al.: Infalling groups and galaxy transformations in the cluster A2142
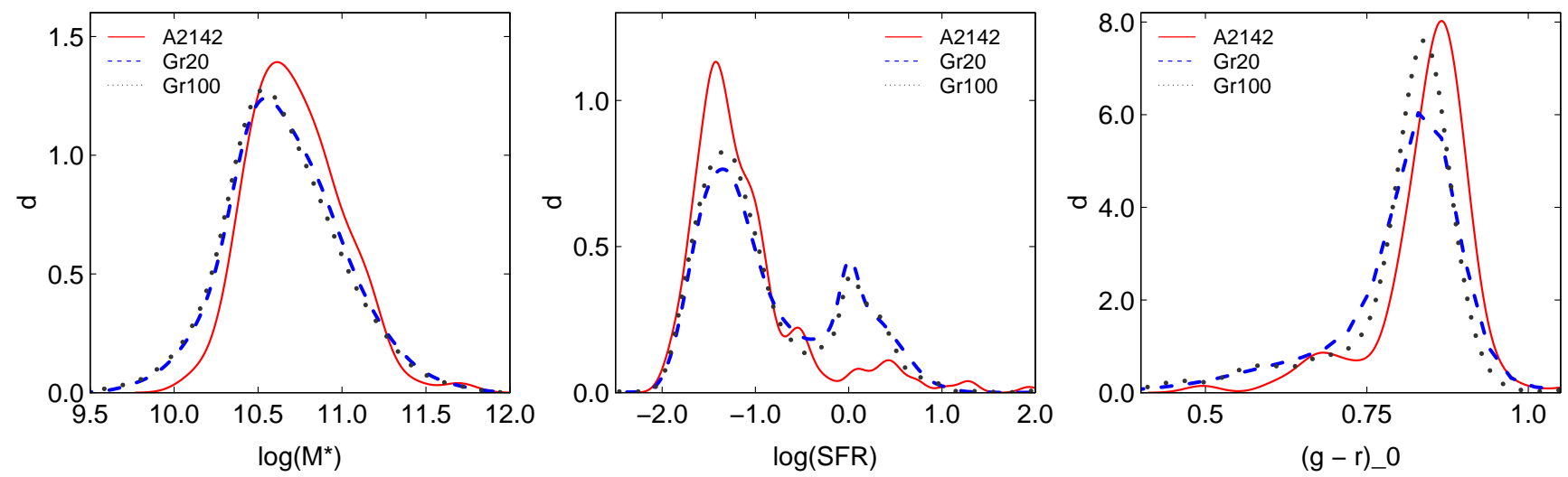

Fig. 13. Distributions of stellar masses (left panel), star formation rates (middle panel), and $(g-r)_{0}$ colours (right panel) of galaxies in the cluster A2142 (red solid line), and in groups with at least 20 and 100 member galaxies (dashed blue and dotted grey lines, correspondingly) in the distance interval 225-280 $\mathrm{h}^{-1} \mathrm{Mpc}$ from the group catalogue by Tempel et al. (2014b), for galaxies brighter than the completeness limit of A2142, $M_{r}=-19.6+5 \log _{10} h$.

brightest galaxy of an infalling group or cluster. We can use several approaches to estimate the mass of a possible merging group. One possibility is to use the median mass estimate of poor groups of galaxies in the Tempel et al. (2014b) catalogue, $M_{\mathrm{gr}} \approx 10^{13} \mathrm{~h}^{-1} M_{\odot}$. Assuming that this galaxy is the brightest galaxy in a group or cluster we can estimate its mass using the relation between the stellar mass $M *$ of a galaxy and its halo mass $M_{\text {halo }}$ from Moster et al. (2010),

$\frac{M_{*}}{M_{\text {halo }}}=2\left(\frac{M_{*}}{M_{\text {halo }}}\right)_{0}\left[\left(\frac{M_{\text {halo }}}{M_{1}}\right)^{-\beta}+\left(\frac{M_{\text {halo }}}{M_{1}}\right)^{\gamma}\right]^{-1}$,

where $\left(M_{*} / M_{\text {halo }}\right)_{0}=0.02817$ is the normalisation of the stellar to halo mass relation, the halo mass $M_{\text {halo }}$ is the virial mass of haloes, $M_{1}=7.925 \times 10^{11} M_{\odot}$ is a characteristic mass, and $\beta=1.068$ and $\gamma=0.611$ are the slopes of the low- and highmass ends of the relation, respectively.

Both BCGs have stellar masses of about $M_{*} \approx$ $5 \times 10^{11} \mathrm{~h}^{-1} M_{\odot}$, and we obtain that they could have haloes with mass of the order of $M_{\text {halo }} \approx 10^{14} \mathrm{~h}^{-1} M_{\odot}$. This is lower mass limit only and the actual halo masses may be higher (Moster et al. 2017). Several mass estimates for the cluster A2142 give mass of the order of $M \approx 10^{15} \mathrm{~h}^{-1} M_{\odot}$ (Munari et al. 2014; Einasto et al. 2015). Therefore the range of mass ratios of the infalling group and main cluster from different estimates is wide. However, our estimates are of the same order as obtained in Rossetti et al. (2013) who mention that the present data do not yet constrain this mass very strongly. We note that galaxies from the possible BCG2 group have stellar populations characteristic of the central parts of rich clusters, supporting the possibility that they belonged to the rich cluster before merging.

$G_{\mathrm{E}}$ group. In Fig. 5 we identified galaxies located at the edge of the infalling group $G_{\mathrm{E}}$, which is described in Eckert et al. (2014, 2017). Infall of the group affects its gas and may induce recent star formation activity in galaxies that are now observed as recently quenched and have young stellar populations with stellar ages of approximately 2 Gyr (Fig. 10). This coincides with the estimate of a timescale of a mixing of the infalling group gas with the surrounding intracluster medium (Eckert et al. 2017). In the PPS diagram, galaxies from this group lie at early infall region with infall time larger than 1 Gyr (Fig. 9), which agrees with the timescale for infall. Typical mass of poor groups of galaxies is of the order of $M_{\mathrm{gr}} \approx 10^{13} \mathrm{~h}^{-1} M_{\odot}$; this coincides with the mass estimation for this group by Eckert et al. (2014).
C3 group. The poorest component in A2142 may represent galaxy group infalling to the main cluster along the supercluster axis. The presence of star-forming galaxies in this group may be an indication of star formation induced by infall. Einasto et al. (2015) found that its mass is of the order of $M \approx 1.5 \times 10^{14} \mathrm{~h}^{-1} M_{\odot}$. If we estimate its mass using the stellar mass of its brightest galaxy we get a mass estimate of the group of approximately $M \approx 1 \times 10^{14} \mathrm{~h}^{-1} M_{\odot}$, which is comparable to the mass estimate for the BCG2 group.

Subclusters M1-M3. The mass of the cluster A2142 outskirts component $C 2$ (all three subclusters together) is approximately $M_{\mathrm{C} 2} \approx 2 \times 10^{14} \mathrm{~h}^{-1} M_{\odot}$ (Table 1 and Einasto et al. 2015). To estimate masses of individual subclusters, we cannot apply the relation between stellar mass and halo mass, as above for the possible BCG groups, since this relation is derived for the central galaxies in virialised haloes (Moster et al. 2010). Therefore we used a very simple mass estimate. Stellar mass in galaxies form approximately $1 \%$ of the supercluster mass in which they are embedded (Einasto et al. 2015, 2016). If we consider that the stellar mass of galaxies in subclusters M1-M3 gives $1 \%$ of their total mass, then we obtain from the sum of stellar masses of galaxies in subclusters that the mass of the subclusters M1 and M2 is $M \approx 0.9 \times 10^{14} \mathrm{~h}^{-1} M_{\odot}$ and the mass of the subcluster M3 is $M \approx 1.5 \times 10^{14} \mathrm{~h}^{-1} M_{\odot}$. For the full outskirts region these estimates give mass on the same order as the estimate for $C 2$ in Einasto et al. (2015).

Together these estimates give the total mass in infalling groups and subclusters approximately $M \approx 6 \times 10^{14} \mathrm{~h}^{-1} M_{\odot}$. Kim et al. (2015) predicted from the analysis of the mass growth of massive haloes in Horizon Run 4 simulations that haloes with mass of the order of $10^{15} \mathrm{~h}^{-1} M_{\odot}$ at redshift $z=0$, similar to cluster A2142, have gathered half of their mass during last 4 Gyr. Therefore the total mass in infalling subclusters is enough for the mass growth of A2142 during this period. However, simulations also predict that up to $40 \%$ of mass growth of haloes comes from smooth accretion of dark matter that was never bound in smaller haloes (Genel et al. 2010; Haines et al. 2017b). This suggests that we may have overestimated the mass of infalling subclusters.

At the same time, A2142 embeds a surprisingly large number of luminous galaxies for its richness (Fig. 12). A large number of luminous galaxies may come from a formation of the cluster by merging two or more rich groups and clusters. The magnitude gap of the brightest galaxies in clusters is one indicator of 
the time since the last major merger (Mulroy et al. 2017, and references therein). In A2142, the BCG2 is associated with the infalling group; the last major merger may be related to the third brightest galaxy, BCG3. In A2142 the magnitude gap between the BCG1 and BCG3 in $r$-band is one magnitude, the BCG3 lies at the projected clustercentric distance of $D_{\mathrm{c}} \approx 0.45 \mathrm{~h}^{-1} \mathrm{Mpc}$. This is in agreement with the suggestion that the time since the last major merger in A2142 may be at least 4 Gyr (Deason et al. 2013; Mulroy et al. 2017).

This prediction supports the suggestion that the possible major merger of rich clusters, which could be the A2142 progenitors, could have happened not later than $4 \mathrm{Gyr}$ ago. In the PPS diagram galaxies with infall time of the order of 4 Gyr occupy an early infall region with clustercentric distances less than $0.5 \mathrm{~h}^{-1} \mathrm{Mpc}$ (Haines et al. 2015). In A2142 this region is populated by galaxies with very old stellar populations with median age of about 10-11 Gyr. This suggests that galaxy populations in the central region of the cluster may belong to the progenitors of A2142 cluster before accreting. In addition, Martel et al. (2014) used simulations to show that the brightest galaxies with large peculiar velocities are associated with major mergers between clusters. If mergers of clusters took place at latest at redhifts $z \geq 0.3$, then clusters had time to reach equilibrium at present. However, if clusters are constantly disturbed by mergers they may not reach equilibrium, as in the case of A2142 where we are witnessing ongoing or recent mergers by groups and subclusters of galaxies causing gas sloshing in the cluster.

The presence of substructure in A2142 may influence mass estimations, but Old et al. (2018) have showed that although the mass estimates of galaxy clusters may be biased in the presence of substructures, the most massive clusters are largely unaffected. Also, various mass estimates of A2142 agree well (Munari et al. 2014; Einasto et al. 2015; Eckert et al. 2017). Therefore, such mergers may explain the richness of A2142 but the discrepancy of mass remains.

\section{Summary}

The analysis of the structure and galaxy populations in the cluster A2142 and its outskirts revealed several infalling groups and subclusters and showed a rapid change in galaxy properties in the infall region of the cluster at clustercentric distances 1.6-2.2 $\mathrm{h}^{-1} \mathrm{Mpc}$. The central, virialised region of the cluster at clustercentric distances $D_{\mathrm{c}}<0.5 \mathrm{~h}^{-1} \mathrm{Mpc}$ is populated with galaxies with old stellar population that have ages of 10-11 Gyr. Even in this region the properties of galaxies are affected by recent mergers. With the increase of the clustercentric distance the properties of galaxies change and in the infall region of the cluster at $D_{\mathrm{c}} \approx 1.6-1.8 \mathrm{~h}^{-1} \mathrm{Mpc}$ median stellar ages of galaxies are approximately 2 Gyr only. Most star-forming or recently quenched galaxies lie in this region. At higher clustercentric distances $\left(D_{\mathrm{c}}>2.25 \mathrm{~h}^{-1} \mathrm{Mpc}\right)$ the median age of galaxies is approximately $8 \mathrm{Gyr}$, which is lower than in the cluster centre (although the scatter in ages is large). At this distance interval star-forming galaxies lie in subcluster M2.

Infalling groups and subclusters have different galaxy contents. One of these (M1) may cause radio ridge of A2142. Cluster BCGs, subcluster M3, and the cluster itself are aligned along the supercluster axis. Ongoing mergers by infalling groups and subclusters give rise to the X-ray and radio structures of the cluster and affect the properties of galaxies in the cluster. We speculate that A2142 has been formed through merging and accretion of other groups and clusters at least 4 Gyr ago along the supercluster axis where the accretion of groups may be enhanced due to high overall density. Subcluster M3, infalling along the supercluster axis, may represent another merging cluster along the supercluster axis. The estimated total mass in infalling groups and subclusters, $M \approx 6 \times 10^{14} \mathrm{~h}^{-1} M_{\odot}$. To be consistent with the predicted mass growth of haloes with mass of the order of $10^{15} \mathrm{~h}^{-1} M_{\odot}$ from redshift $z=0.5$ (half-mass epoch) to the present in simulations we assume that we may have overestimated the mass in subclusters. Stellar masses of galaxies in A2142 are, on average, higher, their star formation rates are lower, and colours are redder than those in other groups and clusters. The richness of the cluster A2142 is also higher than the richness of other rich clusters of the same mass. The reasons for these differences are not yet clear. Clarifying this may lead to better understanding about the formation and evolution of rich galaxy clusters and the properties of dark matter.

In a forthcoming work, we analyse the whole SCl A2142, its galaxy, group, and filament populations in the collapsing core and in outer regions to study the complex evolution of galaxies in the cluster and in the whole supercluster. We also continue to study rich, merging galaxy clusters and their galaxy populations in collapsing cores of superclusters to understand better their coevolution within the cosmic web.

Acknowledgements. We thank the referee for suggestions and comments that helped improve the paper. We are pleased to thank the SDSS Team for the publicly available data releases. Funding for the Sloan Digital Sky Survey (SDSS) and SDSS-II has been provided by the Alfred P. Sloan Foundation, the Participating Institutions, the National Science Foundation, the U.S. Department of Energy, the National Aeronautics and Space Administration, the Japanese Monbukagakusho, and the Max Planck Society, and the Higher Education Funding Council for England. The SDSS website is http://www. sdss.org/. The SDSS is managed by the Astrophysical Research Consortium (ARC) for the Participating Institutions. The Participating Institutions are the American Museum of Natural History, Astrophysical Institute Potsdam, University of Basel, University of Cambridge, Case Western Reserve University, The University of Chicago, Drexel University, Fermilab, the Institute for Advanced Study, the Japan Participation Group, The Johns Hopkins University, the Joint Institute for Nuclear Astrophysics, the Kavli Institute for Particle Astrophysics and Cosmology, the Korean Scientist Group, the Chinese Academy of Sciences (LAMOST), Los Alamos National Laboratory, the Max-Planck-Institute for Astronomy (MPIA), the Max-Planck-Institute for Astrophysics (MPA), New Mexico State University, Ohio State University, University of Pittsburgh, University of Portsmouth, Princeton University, the United States Naval Observatory, and the University of Washington. The present study was supported by the ETAG projects IUT26-2 and IUT40-2, and by the European Structural Funds grant for the Centre of Excellence "Dark Matter in (Astro)particle Physics and Cosmology" TK133. HL is funded by PUT1627 grant from Estonian Research Council. This work has also been supported by ICRAnet through a professorship for Jaan Einasto. We thank Prof. Juhan Kim for comments and Jukka Nevalainen for inspiring discussions.

\section{References}

Agulli, I., Aguerri, J. A. L., Diaferio, A., Dominguez Palmero, L., \& Sánchez-Janssen, R. 2017, MNRAS, 467, 4410

Ahn, C. P., Alexandroff, R., Allende Prieto, C., et al. 2014, ApJS, 211, 17

Aihara, H., Allende Prieto, C., An, D., et al. 2011, ApJS, 193, 29

Balogh, M. L., Morris, S. L., Yee, H. K. C., Carlberg, R. G., \& Ellingson, E. 1999, ApJ, 527, 54

Baxter, E., Chang, C., Jain, B., et al. 2017, ApJ, 841, 18

Bezanson, R., van Dokkum, P., \& Franx, M. 2012, ApJ, 760, 62

Blanton, M. R., \& Roweis, S. 2007, AJ, 133, 734

Blanton, M. R., Hogg, D. W., Bahcall, N. A., et al. 2003, ApJ, 592, 819

Bond, J. R., Kofman, L., \& Pogosyan, D. 1996, Nature, 380, 603

Brinchmann, J., Charlot, S., White, S. D. M., et al. 2004, MNRAS, 351, 1151

Bruzual, G., \& Charlot, S. 2003, MNRAS, 344, 1000

Cappellari, M., \& Emsellem, E. 2004, PASP, 116, 138

Chon, G., Böhringer, H., \& Zaroubi, S. 2015, A\&A, 575, L14

Cohen, S. A., Hickox, R. C., Wegner, G. A., Einasto, M., \& Vennik, J. 2014, ApJ, 783, 136 
Cohen, S. A., Hickox, R. C., Wegner, G. A., Einasto, M., \& Vennik, J. 2017, ApJ, 835, 56

Costa-Duarte, M. V., Sodré, L., \& Durret, F. 2013, MNRAS, 428, 906 Deason, A. J., Conroy, C., Wetzel, A. R., \& Tinker, J. L. 2013, ApJ, 777, 154 Deshev, B., Finoguenov, A., Verdugo, M., et al. 2017, A\&A, 607, A131 Eckert, D., Molendi, S., Owers, M., et al. 2014, A\&A, 570, A119 Eckert, D., Gaspari, M., Owers, M. S., et al. 2017, A\&A, 605, A25

Einasto, M., Saar, E., Martínez, V. J., et al. 2008, ApJ, 685, 83

Einasto, M., Tago, E., Saar, E., et al. 2010, A\&A, 522, A92

Einasto, M., Liivamägi, L. J., Tago, E., et al. 2011, A\&A, 532, A5

Einasto, M., Vennik, J., Nurmi, P., et al. 2012, A\&A, 540, A123

Einasto, M., Lietzen, H., Tempel, E., et al. 2014, A\&A, 562, A87

Einasto, M., Gramann, M., Saar, E., et al. 2015, A\&A, 580, A69

Einasto, M., Lietzen, H., Gramann, M., et al. 2016, A\&A, 595, A70

Farnsworth, D., Rudnick, L., Brown, S., \& Brunetti, G. 2013, ApJ, 779, 189

Foëx, G., Chon, G., \& Böhringer, H. 2017, A\&A, 601, A145

Forman, W., Markevitch, M., Jones, C., Vikhlinin, A., \& Churazov, E. 2001, in Clusters of Galaxies and the High Redshift Universe Observed in X-rays, eds. D. M. Neumann, \& J. T. V. Tran, 33

Fraley, C., \& Raftery, A. E. 2006, Technical Report, Dep. of Statistics, University of Washington, 504, 1

Genel, S., Bouché, N., Naab, T., Sternberg, A., \& Genzel, R. 2010, ApJ, 719, 229

Girardi, M., Barrena, R., Boschin, W., \& Ellingson, E. 2008, A\&A, 491, 379

Govoni, F., Dolag, K., Murgia, M., et al. 2010, A\&A, 522, A105

Gramann, M., Einasto, M., Heinämäki, P., et al. 2015, A\&A, 581, A135

Haines, C. P., Pereira, M. J., Smith, G. P., et al. 2015, ApJ, 806, 101

Haines, C. P., Finoguenov, A., Smith, G. P., et al. 2017a, MNRAS, submitted, ArXiv e-prints [arXiv: 1709.04945]

Haines, C. P., Iovino, A., Krywult, J., et al. 2017b, A\&A, 605, A4

Hirv, A., Pelt, J., Saar, E., et al. 2017, A\&A, 599, A31

Hopkins, P. F., Bahcall, N. A., \& Bode, P. 2005, ApJ, 618, 1

Huchra, J. P., \& Geller, M. J. 1982, ApJ, 257, 423

Ihaka, R., \& Gentleman, R. 1996, J. Comput. Graph. Stat., 5, 299

Jaffé, Y. L., Smith, R., Candlish, G. N., et al. 2015, MNRAS, 448, 1715

Jõeveer, M., \& Einasto, J. 1978, in Large Scale Structures in the Universe, eds. M. S. Longair \& J. Einasto, IAU Symp., 79, 241

Jõeveer, M., Einasto, J., \& Tago, E. 1978, MNRAS, 185, 357

Johnston-Hollitt, M., Sato, M., Gill, J. A., Fleenor, M. C., \& Brick, A.-M. 2008, MNRAS, 390, 289

Kauffmann, G., Heckman, T. M., White, S. D. M., et al. 2003a, MNRAS, 341, 33

Kauffmann, G., Heckman, T. M., White, S. D. M., et al. 2003b, MNRAS, 341, 54

Kim, J., Park, C., L'Huillier, B., \& Hong, S. E. 2015, J. Korean Astron. Society, 48,213

Kofman, L. A., \& Shandarin, S. F. 1988, Nature, 334, 129

Komatsu, E., Smith, K. M., Dunkley, J., et al. 2011, ApJS, 192, 18

Krause, M. O., Ribeiro, A. L. B., \& Lopes, P. A. A. 2013, A\&A, 551, A143

Kravtsov, A. V., \& Borgani, S. 2012, ARA\&A, 50, 353

Lietzen, H., Tempel, E., Heinämäki, P., et al. 2012, A\&A, 545, A104

Liivamägi, L. J., Tempel, E., \& Saar, E. 2012, A\&A, 539, A80

Liu, A., Yu, H., Tozzi, P., \& Zhu, Z.-H. 2016, ApJ, 821, 29

Luparello, H. E., Lares, M., Yaryura, C. Y., et al. 2013, MNRAS, 432, 1367

Mahajan, S., Raychaudhury, S., \& Pimbblet, K. A. 2012, MNRAS, 427, 1252

Mahdavi, A., Hoekstra, H., Babul, A., Balam, D. D., \& Capak, P. L. 2007, ApJ, 668,806

Maraston, C., Strömbäck, G., Thomas, D., Wake, D. A., \& Nichol, R. C. 2009, MNRAS, 394, L107

Markevitch, M., Ponman, T. J., Nulsen, P. E. J., et al. 2000, ApJ, 541, 542

Martel, H., Robichaud, F., \& Barai, P. 2014, ApJ, 786, 79
Martínez, V. J., \& Saar, E. 2002, Statistics of the Galaxy Distribution (Boca Raton: Chapman \& Hall/CRC)

McIntosh, M., de Propris, R., \& West, M. 2016, American Astronomical Society Meeting Abstracts, 227, 235.18

Merluzzi, P., Busarello, G., Haines, C. P., et al. 2015, MNRAS, 446, 803

Molnar, S. M., Hearn, N., Haiman, Z., et al. 2009, ApJ, 696, 1640

Moster, B. P., Somerville, R. S., Maulbetsch, C., et al. 2010, ApJ, 710, 903

Moster, B. P., Naab, T., \& White, S. D. M. 2017, ArXiv e-prints [arXiv: 1705.05373$]$

Mulroy, S. L., McGee, S. L., Gillman, S., et al. 2017, MNRAS, 472, 3246

Munari, E., Biviano, A., \& Mamon, G. A. 2014, A\&A, 566, A68

Munari, E., Grillo, C., De Lucia, G., et al. 2016, ApJ, 827, L5

Muzzin, A., van der Burg, R. F. J., McGee, S. L., et al. 2014, ApJ, 796, 65

Okabe, N., \& Umetsu, K. 2008, PASJ, 60, 345

Old, L., Wojtak, R., Pearce, F. R., et al. 2018, MNRAS, 475, 853

Oman, K. A., Hudson, M. J., \& Behroozi, P. S. 2013, MNRAS, 431, 2307

O’Mill, A. L., Proust, D., Capelato, H. V., et al. 2015, MNRAS, 453, 868

Owers, M. S., Nulsen, P. E. J., \& Couch, W. J. 2011, ApJ, 741, 122

Paccagnella, A., Vulcani, B., Poggianti, B. M., et al. 2016, ApJ, 816, L25

Paccagnella, A., Vulcani, B., Poggianti, B. M., et al. 2017, ApJ, 838, 148

Park, C., \& Hwang, H. S. 2009, ApJ, 699, 1595

Paz, D. J., Sgró, M. A., Merchán, M., \& Padilla, N. 2011, MNRAS, 414, 2029

Pearson, D. W., Batiste, M., \& Batuski, D. J. 2014, MNRAS, 441, 1601

Plionis, M., \& Basilakos, S. 2002, MNRAS, 329, L47

Porter, S. C., Raychaudhury, S., Pimbblet, K. A., \& Drinkwater, M. J. 2008, MNRAS, 388, 1152

Proust, D., Quintana, H., Carrasco, E. R., et al. 2006, A\&A, 447, 133

Reisenegger, A., Quintana, H., Carrasco, E. R., \& Maze, J. 2000, AJ, 120, 523

Rhee, J., Smith, R., Choi, H., et al. 2017, ApJ, 843, 128

Rossetti, M., Eckert, D., De Grandi, S., et al. 2013, A\&A, 556, A44

Salim, S., Rich, R. M., Charlot, S., et al. 2007, ApJS, 173, 267

Sarzi, M., Falcón-Barroso, J., Davies, R. L., et al. 2006, MNRAS, 366, 1151

Shankar, F., Bernardi, M., \& Haiman, Z. 2009, ApJ, 694, 867

Small, T. A., Ma, C.-P., Sargent, W. L. W., \& Hamilton, D. 1998, ApJ, 492, 45

Song, H., Hwang, H. S., Park, C., \& Tamura, T. 2017, ApJ, 842, 88

Suhhonenko, I., Einasto, J., Liivamägi, L. J., et al. 2011, A\&A, 531, A149

Tchernin, C., Eckert, D., Ettori, S., et al. 2016, A\&A, 595, A42

Tempel, E., \& Tamm, A. 2015, A\&A, 576, L5

Tempel, E., Tago, E., \& Liivamägi, L. J. 2012, A\&A, 540, A106

Tempel, E., Stoica, R. S., Martínez, V. J., et al. 2014a, MNRAS, 438, 3465

Tempel, E., Tamm, A., Gramann, M., et al. 2014b, A\&A, 566, A1

Tempel, E., Guo, Q., Kipper, R., \& Libeskind, N. I. 2015, MNRAS, 450, 2727

Tempel, E., Stoica, R. S., Kipper, R., \& Saar, E. 2016, Astron. Computing, 16 17

Tremonti, C. A., Heckman, T. M., Kauffmann, G., et al. 2004, ApJ, 613, 898 van de Weygaert, R., \& Schaap, W. 2009, in Data Analysis in Cosmology, eds. V. J. Martínez, E. Saar, E. Martínez-González, \& M.-J. Pons-Bordería (Berlin: Springer Verlag), Lect. Not. Phys., 665, 291

Venturi, T., Rossetti, M., Brunetti, G., et al. 2017, A\&A, 603, A125

Vijayaraghavan, R., \& Ricker, P. M. 2013, MNRAS, 435, 2713

Weinzirl, T., Aragón-Salamanca, A., Gray, M. E., et al. 2017, MNRAS, 471, 182

West, M. J., \& Blakeslee, J. P. 2000, ApJ, 543, L27

West, M. J., de Propris, R., Bremer, M. N., \& Phillipps, S. 2017, Nat. Astron., 1, 0157

Wittman, D., Golovich, N., \& Dawson, W. A. 2017, ApJ, submitted, ArXiv eprints [arXiv: 1701.05877]

Yoon, H., Chung, A., Smith, R., \& Jaffé, Y. L. 2017, ApJ, 838, 81

Zeldovich, I. B., Einasto, J., \& Shandarin, S. F. 1982, Nature, 300, 407 